\title{
OPTIMAL CONTROL OF THE STATIONARY QUANTUM DRIFT-DIFFUSION MODEL*
}

\author{
A. UNTERREITER ${ }^{\dagger}$ AND S. VOLKWEIN ${ }^{\ddagger}$
}

\begin{abstract}
In this work an optimal control problem for a stationary quantum drift diffusion (QDD) model is analyzed. This QDD model contains four space-dependent observables: The nonnegative particle density of electrons, the electrostatic potential, the quantum quasi-Fermi potential and the current density. The goal is to optimize the shape of quantum barriers in a quantum diode. Existence of optimal solutions is proved. Moreover, first-order necessary optimality conditions are
\end{abstract} derived.

Key words. Optimal control, quantum drift diffusion model, optimality conditions.

Subject classifications. 35J55, 49J20, 49K20.

\section{Introduction}

Ultra-small semiconductor devices exhibit electronic performances relying intrinsically on quantum-mechanical effects. In particular, in certain switching situations the electric current flowing through the device depends in a non-monotone way on the applied voltage. This important phenomenon is known as negative differential resistance (NDR) effect. Aside from finding tractable mathematical models for NDR effects, the question of optimizing the system's parameters with respect to desired performances has raised considerable attention in the literature; see, e.g., in $[4,6,7,12]$ for optimal control of a drift-diffusion model. Furthermore, we refer to [8], where an inverse problem for semiconductor equations was considered that arises in modelling a laser beam induced current (LBIC). For engineering aspects we refer to [15, 16, 17].

In this work we want to optimize the shape of quantum barriers in a quantum diode to achieve for a given external voltage $V_{D}$ an optimal current $I_{d}$. The quantum diode consists of several sandwiched crystals of different ground energy levels whose gaps are described by the quantum barrier function $B$. The quantum diode's performance is overall determined by the location, the width and the ground energy values of the involved crystals. While the crystals' locations are more or less limited by technological possibilities, their length and their quality (thus, their ground energy's value) are variable. In particular, one can optimize length and quality to get as close as possible to a desired IV-curve.

It is the present paper's plan to investigate a mathematical model for this optimization procedure. The goal of the optimization is to determine amongst a class of admissible barrier functions the optimal $B$ to achieve a desired IV-curve. We suppose the set of admissible barrier functions to be parameterized by $\ell$ variables ranging in the set of admissible controls $U_{\mathrm{ad}}$.

The paper is organized in the following manner: In Section 2 we introduce the stationary quantum drift diffusion (QDD) model, prove existence of $H^{1}$ solutions and derive a-priori bounds. The optimal control problem is formulated in Section 3. Existence of optimal solutions is shown utilizing the a-priori bounds derived in Section 3.

\footnotetext{
${ }^{*}$ Received: June 20, 2006; accepted (in revised version): November 16, 2006. Communicated by Pierre Degond.

${ }^{\dagger}$ Technische Universität Berlin, Fakultät II - Mathematik und Naturwissenschaften, MA 6-3, Straße des 17. Juni 136, D - 10623 Berlin, Germany (unterreiter@math.tu-berlin.de).

¥Institut für Mathematik und Wissenschaftliches Rechnen, Karl-Franzens-Universität Graz, Heinrichstrasse 36, A-8010 Graz, Austria (stefan.volkwein@uni-graz.at).
} 
We present first-order necessary optimality conditions in Section 4.

\section{The quantum drift diffusion model}

The investigations concern a stationary, i.e., time-independent, unipolar QDD model constituted by a system of coupled partial differential equations. The spatial variable $\mathbf{x}$ ranges in the devices' domain $W$. Having in mind realistic shapes for ultra-small devices we assume $W$ to be a cuboid in $d$ space dimensions, $d=1,2$ or $d=3$ :

$$
W=] 0, L_{1}[\times \ldots \times] 0, L_{d}\left[\quad \text { with } \quad L_{1}, \ldots, L_{d}>0 .\right.
$$

Typically the diode's boundary $\Gamma$ splits into a contact region $\Gamma_{D}$ and an insulating region $\Gamma_{N}$. It is quite realistic to assume that $\Gamma_{D}$ consists of two opposing lateral surfaces

$$
\Gamma_{D}=\left\{0, L_{1}\right\} \times\left[0, L_{2}\right] \times \ldots\left[0, L_{d}\right]
$$

while the insulating region is $\Gamma_{N}=\Gamma \backslash \Gamma_{D}$. Obviously, $\Gamma_{N}=\emptyset$ for $d=1$.

2.1. The model equations. In the framework of the stationary QDD model the diode's performance is described by four space-dependent observables: The nonnegative electron's particle density $n$, the electrostatic potential $V$, the quantum quasiFermi potential $F$ and the current density $\vec{J}$. The diode's parameters are the effective mass of electrons $m$, the temperature of the electron gas $T$, the positive, constant mobility of electrons $\mu$, the positive, constant permittivity of the diode's underlying crystal $\varepsilon$, the time-independent, non-negative doping profile $C$, and the time-independent quantum barrier function $B: W \rightarrow \mathbb{R}$. The quantum barrier function $B$ represents the ground energy's gap of one (or more) crystals with respect to a reference crystal. $B$ vanishes in regions, where the reference crystal is present, whereas $B$ does not vanish in a crystal whose ground energy is different from the reference crystal's ground energy. We suppose

$$
B, C \in L^{\infty}(W) .
$$

For the notion of Lebesgue and Sobolev spaces we refer the reader to [1], for instance.

The quantum quasi-Fermi potential $F$ acts as the electron's velocity potential,

$$
\vec{J}=\mu n \nabla F \quad \text { in } W .
$$

Corresponding to the conservation of mass the current density is divergence free:

$$
\nabla \cdot \vec{J}=0 \text { in } W .
$$

The electrostatic potential $V$ is self-consistent, i.e.,

$$
-\epsilon \Delta V=q(n-C) \text { in } W,
$$

where $\epsilon \approx 11.6 \cdot 10^{-12} \frac{\mathrm{As}}{\mathrm{Vm}}$ is the - assumingly: constant — dielectricity constant of the underlying crystal, and the quantum quasi-Fermi potential $F$ is

$$
F=-\frac{\hbar^{2}}{6 m} \frac{\Delta \sqrt{n}}{\sqrt{n}}+k_{B} T \log n+q V+B \quad \text { in } W
$$

where $\hbar \approx 6.626 \cdot 10^{-34} \mathrm{~J} s$ is Planck's constant, $k_{B} \approx 1.38 \cdot 10^{-23} \frac{\mathrm{J}}{\mathrm{K}}$ is Boltzmann's constant and $q \approx 1.6 \cdot 10^{-19}$ As is the elementary charge. The term $-\frac{\hbar^{2}}{6 m} \frac{\Delta \sqrt{n}}{\sqrt{n}}$ is Bohm's 


\begin{tabular}{|l||l|c|}
\hline Quantity & $s$ & Order of magnitude (SI units) \\
\hline $\mathbf{x}$ & $L$ & $7.5 \cdot 10^{-8}$ \\
\hline$n$ & $\bar{C}$ & $10^{24}$ \\
\hline$V$ & $U_{t h}=k_{B} T / q$ & $6.6 \cdot 10^{-3}$ \\
\hline$C$ & $\bar{C}$ & $10^{24}$ \\
\hline$F$ & $k_{B} T$ & $10^{-21}$ \\
\hline$B$ & $k_{B} T$ & $10^{-21}$ \\
\hline$\vec{J}$ & $\mu \bar{C} k_{B} T / L$ & $3.5 \cdot 10^{10}$ \\
\hline
\end{tabular}

TABLE 2.1. Scaling factor $s$ for the involved quantities satisfying Quantity $=s$ times scaled Quantity.

quantum potential; see [2]. The effective electron mass $m$ is typically about one tenth of the electron mass at rest $m \approx 10^{-31} \mathrm{~kg}$; see $[12,13]$.

It is convenient to introduce scaled variables. In Table 2.1 we give the scaling factor $s$ for the involved quantities: Quantity $=s$ times scaled Quantity. Moreover, we use $\mu=2.08 \frac{\mathrm{Am}^{2}}{J}$ and $T=77 \mathrm{~K}$. In what follows we denote the scaled variables with the same symbol as the corresponding unscaled variables. The scaled model equations read

$$
\begin{aligned}
\vec{J} & =n \nabla F & & \text { in } W, \\
\nabla \cdot \vec{J} & =0 & & \text { in } W, \\
-\lambda^{2} \Delta V & =n-C & & \text { in } W, \\
F & =-\varepsilon^{2} \frac{\Delta \sqrt{n}}{\sqrt{n}}+\log (n)+V+B & & \text { in } W,
\end{aligned}
$$

where

$$
0<\varepsilon^{2}=\frac{\hbar^{2}}{6 k_{B} T m L^{2}} \approx 3 \cdot 10^{-3},
$$

and via $\epsilon \approx 11.6 \cdot 10^{-12}$

$$
0<\lambda^{2}=\frac{\epsilon U_{t h}}{q \bar{C} L^{2}} \approx 8.5 \cdot 10^{-4} .
$$

It is convenient to use rather $\rho=\sqrt{n}$ than $n$. Equations (2.1) become

$$
\begin{aligned}
\vec{J} & =\rho^{2} \nabla F & & \text { in } W, \\
\nabla \cdot \vec{J} & =0 & & \text { in } W, \\
-\lambda^{2} \Delta V & =\rho^{2}-C & & \text { in } W, \\
F & =-\varepsilon^{2} \frac{\Delta \rho}{\rho}+\log \left(\rho^{2}\right)+V+B & & \text { in } W .
\end{aligned}
$$

Appropriate boundary conditions for (2.2) are obtained due to the following considerations, where $\nu: \Gamma \rightarrow \mathbb{R}^{d}$ is the outward normal vector: 
a) We wish to optimize the current flowing through the device for a given external voltage $V_{D} . V_{D}$ is applied along the contact region $\Gamma_{D}$, hence

$$
V=V_{D} \quad \text { on } \Gamma_{D}
$$

b) The electron density $\rho^{2}$ along $\Gamma_{D}$ depends on the device and on the surrounding electronic circuit. If $V_{D}$ is "close to the equilibrium value", i.e., $V_{D} \approx 0$, it will be quite reasonable to assume $\rho^{2}$ do be "almost independent of $V_{D}$ ". As a consequence,

$$
\rho=\rho_{D} \quad \text { on } \Gamma_{D} .
$$

Possible choices for $\rho_{D}$ are $\rho_{D}=$ "variational minimum value of an energy functional", see [11], or $\rho_{D}=\sqrt{C}$ leading to vanishing space charge on $\Gamma_{D}$; see [12].

c) In (2.2) the unknown function $F$ can be viewed as a potential for the velocity field for electrons. Hence one has to prescribe $F$ along the contact region $\Gamma_{D}$ where the current enters the device.

$$
F=F_{D} \quad \text { on } \Gamma_{D}
$$

In thermal equilibrium $V_{D}=0$ there is no current at all which canonically leads to $F_{D}=0$ (or another constant). If $V_{D} \neq 0$ then there will a current flow through the device and $F_{D}$ has to be changed. Thus, $F_{D}$ depends on $V_{D}$. A possible choice is $F_{D}=V_{D}$; see [12].

d) Along the insulating region the electric field is expected to have no component orthogonal to the device. Thus

$$
\frac{\partial V}{\partial \nu}=0 \quad \text { on } \Gamma_{N} .
$$

e) Along the insulating region the current density $\vec{J}$ has only tangential components. As $\rho \neq 0$ can be expected we deduce via $\vec{J}=\rho^{2} \nabla F$,

$$
\frac{\partial F}{\partial \nu}=0 \quad \text { on } \Gamma_{N} .
$$

f) The quantum mechanical Bohm potential $-\varepsilon^{2} \frac{\Delta \rho}{\rho}$, the diffusion potential $\log \left(\rho^{2}\right)$, the drift potential $V$ and the barrier potential $B$ all contribute to the velocity field $\nabla F$ and thus determine $\vec{J}$. Aside from the barrier potential $B$, one has to expect that none of these contributions has a component orthogonal to the device. Via d) and e) we deduce $\frac{\partial \log \left(\rho^{2}\right)}{\partial \nu}=0$ on $\Gamma_{N}$. As we expect $\rho \neq 0$, we deduce

$$
\frac{\partial \rho}{\partial \nu}=0 \quad \text { on } \Gamma_{N}
$$


We obtain

$$
\begin{aligned}
-\nabla \cdot\left(\rho^{2} \nabla F\right) & =0 & & \text { in } W, \\
-\varepsilon^{2} \Delta \rho & =\rho\left(F-V-B-\log \left(\rho^{2}\right)\right) & & \text { in } W, \\
-\lambda^{2} \Delta V & =\rho^{2}-C & & \text { in } W, \\
F=F_{D}, \rho & =\rho_{D}, V=V_{D} & & \text { on } \Gamma_{D}, \\
\frac{\partial F}{\partial \nu}=\frac{\partial \rho}{\partial \nu} & =\frac{\partial V}{\partial \nu}=0 & & \text { on } \Gamma_{N} .
\end{aligned}
$$

For the sake of brevity we set

$$
H_{b}=H^{1}(W) \cap L^{\infty}(W),
$$

which is a Banach space supplied with the canonical norm

$$
\|\varphi\|_{b}=\|\varphi\|_{H^{1}(W)}+\|\varphi\|_{L^{\infty}(W)} \quad \text { for } \varphi \in H_{b} .
$$

For $k \in \mathbb{N}$ we set

$$
\left(H_{b}\right)^{k}=\underbrace{H_{b} \times \ldots \times H_{b}}_{k-\text { times }}
$$

endowed with the natural product topology. Recall that in the one-dimensional case $H^{1}(W)$ is continuously embedded in $L^{\infty}(W)$ and we deduce $H_{b}=H^{1}(W) \cap L^{\infty}(W)=$ $H^{1}(W)$. We assume henceforth

$$
F_{D}, \rho_{D}, V_{D} \in H_{b}
$$

and

$$
\underset{\mathbf{x} \in W}{\operatorname{essinf}} \rho_{D}(\mathbf{x})>0 .
$$

In (2.3) the square of the electron density $\rho=\rho(\mathbf{x}) \geq 0$, the quantum quasi-Fermi level $F=F(\mathbf{x})$, and the electric potential $V=V(\mathbf{x})$ are unknowns. The current density is given by $\vec{J}=\rho^{2} \nabla F$.

Equations (2.3) possibly have several solutions. Existence of weak solutions in $H^{1}$ - shorthanded as " $H^{1}$ solutions" - is established in $[3,13,18] . H^{1}$ solutions of $(2.3)$ belong to the Sobolev space

$$
H_{\circ}=H_{0}^{1}\left(W \cup \Gamma_{N}\right)=\left\{\varphi \in H^{1}(W) \mid \varphi=0 \text { on } \Gamma_{D}\right\} .
$$

Since $\Gamma_{D}$ consists of two opposing lateral surfaces the Sobolev space $H_{\circ}$ allows for a Poincaré inequality: there exists a constant $C_{P}>0$ such that

$$
\int_{W} \varphi^{2} \mathrm{~d} \mathbf{x} \leq C_{P} \int_{W}|\nabla \varphi|^{2} \mathrm{~d} \mathbf{x} \quad \text { for all } \varphi \in H_{\circ} .
$$

As a consequence, the bilinear form

$$
\langle\varphi, \psi\rangle_{H_{\circ}}=\int_{W} \nabla \varphi \cdot \nabla \psi \mathrm{d} \mathbf{x} \quad \text { for } \varphi, \psi \in H_{0}^{1}(W)
$$

is an inner product in $H_{\circ}$ and $\left(H_{\circ},\langle\cdot, \cdot\rangle_{H_{\circ}}\right)$ is a Hilbert space.

Definition 2.1. The triple $(F, \rho, V)$ is a $H^{1}$ solution of (2.3) if and only if 
1) $F-V_{D}, \rho-\rho_{D}, V-V_{D} \in H_{\circ} ; F \in L^{\infty}(W)$;

2) $\rho \geq 0$ a.e. in $W$;

3) for all $\varphi \in H_{\circ}$,

$$
\begin{aligned}
& \int_{W} \rho^{2} \nabla F \cdot \nabla \varphi \mathrm{d} \mathbf{x}=0 \\
& \int_{W} \varepsilon^{2} \nabla \rho \cdot \nabla \varphi \mathrm{d} \mathbf{x}=\int_{W} \rho\left(F-V-B-\log \left(\rho^{2}\right)\right) \varphi \mathrm{d} \mathbf{x}, \\
& \int_{W} \lambda^{2} \nabla V \cdot \nabla \varphi \mathrm{d} \mathbf{x}=\int_{W}\left(\rho^{2}-C\right) \varphi \mathrm{d} \mathbf{x}
\end{aligned}
$$

where in $(2.5 \mathrm{~b})$ and in the sequel

$$
0 \cdot \log \left(0^{2}\right)=0^{2} \cdot \log \left(0^{2}\right)=0 .
$$

REMARK 2.2. Since the space dimension $d$ does not exceed 3 a variational formulation characterizing $\rho$ is available: $\rho$ is the minimizer of

$$
E(\varrho)=E_{q u}(\varrho)+E_{e l}(\varrho)+\int_{W} \varrho^{2}(B-F) \mathrm{d} \mathbf{x}
$$

- if $d \leq 3$ we have $\forall \rho, F \in H^{1}(W): \rho^{2} F \in L^{1}(W)$ ensuring directional derivatives of $E$ exist in all directions $\phi \in H^{1}$ such that we indeed can pass for the minimizer of $E$ to $(2.5 \mathrm{~b})$ - in the $H^{1}(W)$-weakly closed, convex set $\rho_{D}+H_{\circ}$ (see [3]), where

$$
E_{q u}(\rho)=\varepsilon^{2} \int_{W}|\nabla \rho|^{2} \mathrm{~d} \mathbf{x}+\int_{W} \rho^{2}\left(\log \left(\rho^{2}\right)-1\right) \mathrm{d} \mathbf{x}
$$

is the quantum internal energy, i.e., Bohm's interaction energy plus the classical internal energy,

$$
E_{e l}(\varrho)=\frac{1}{2} \int_{W}\left|\nabla V_{\circ}\right|^{2} \mathrm{~d} \mathbf{x}+\int_{W} \rho^{2} V^{*} \mathrm{~d} \mathbf{x},
$$

is the electrostatic energy, where $V_{\circ}$ solves

$$
-\lambda^{2} \Delta V_{\circ}=\varrho^{2}-C, \quad V_{\circ} \in H_{\circ},
$$

uniquely in $H_{\circ}$ and $V^{*}$ solves

$$
-\lambda^{2} \Delta V^{*}=0, \quad V^{*}-V_{D} \in H_{\circ},
$$

uniquely in $H^{1}(W)$, i.e., the function $V^{*}$ is the Laplace-extension of the boundary data $V_{D}$.

REMARK 2.3.

a) The functional $E$ has at most one minimizer in $\rho_{D}+H_{\circ}$; see [11].

b) In Definition 2.1 we require $F \in L^{\infty}(W)$. Due to the maximum principle one can deduce $F \in L^{\infty}(W)$ from (2.5a) via $F_{D} \in L^{\infty}(W)$ - provided one has a priori estimates ensuring $\inf _{W} \rho>0$. However, available proofs of $\inf _{W} \rho>0$ in turn rely on $F \in L^{\infty}(W)$. So by requiring $F \in L^{\infty}(W)$ we do not want to make things unnecessarily sophisticated here. 
c) Due to available results the assumption $F^{\infty}(W)$ is seemingly not very restrictive: up to now is not known whether (2.3) has $H^{1}$ solutions $F \notin L^{\infty}(W)$.

d) With the same notations as in Definition 2.1-4) the function $V=V_{\circ}+V^{*}$ is the unique $H^{1}(W)$-weak solution of

$$
-\lambda^{2} \Delta V=\rho^{2}-C, \quad V-V_{D} \in H_{\circ} .
$$

Furthermore, for each triple $(C, F, B) \in\left(L^{\infty}(W)\right)^{3}$ and each $\rho$ minimizing $E$ in $\rho_{D}+H_{\circ}$, the pair $(\rho, V)$ satisfies $(2.5 \mathrm{~b}),(2.5 \mathrm{c})$.

e) Concerning the electrostatic energy we deduce via Poincaré's inequality

$$
\begin{aligned}
& \frac{1}{2} \int_{W}\left|\nabla V_{\circ}\left(\rho^{2}-C\right)\right|^{2} \mathrm{~d} \mathbf{x} \\
& \quad \leq \frac{C_{P} \operatorname{meas}(W)}{2 \lambda^{4}}\left(\|\rho\|_{L^{\infty}(W)}^{2}+\|C\|_{L^{\infty}(W)}\right)^{2} \quad \text { for all } \rho \in L^{\infty}(W),
\end{aligned}
$$

where here and in the sequel "meas" is the Lebesgue measure.

Now we establish the existence of a $H^{1}$ solution and a-priori estimates. The proof can be found in the appendix.

Theorem 2.4. Let (A1)-(A5) and

$$
\varepsilon, \lambda>0
$$

hold. Then (2.3) has a $H^{1}$ solution and there is a constant $C>0$ depending on $W, \varepsilon$, $\lambda,\left\|\rho_{D}\right\|_{b},\left\|V_{D}\right\|_{b},\|C\|_{L^{\infty}(W)},\left\|F_{D}\right\|_{b}$, such that for each $H^{1}$ solution $(F, \rho, V)$,

$$
\|F\|_{b}+\|\rho\|_{b}+\|V\|_{b} \leq C e^{C e^{\|B\|_{L} \infty(W)}} .
$$

2.2. The current. The Dirichlet boundary $\Gamma_{D}$ splits into two (disjoint) parts

$$
\Gamma_{D}^{1}=\{0\} \times W_{D} \quad \text { and } \quad \Gamma_{D}^{2}=\left\{L_{1}\right\} \times W_{D}
$$

with $\left.W_{D}=\right] 0, L_{2}[\times \ldots \times] 0, L_{d}\left[\right.$. The current $I_{i}(F, \rho)$ flowing through $\Gamma_{D}^{i}, i=1$ or $i=2$, is given by

$$
\begin{aligned}
I_{i}(F, \rho) & =(-1)^{i} q \int_{W_{D}}\left(\vec{J} \cdot \vec{e}_{1}\right)\left(z_{i}, x_{2}, \ldots, x_{d}\right) \mathrm{d}\left(x_{2}, \ldots, x_{d}\right) \\
& =(-1)^{i} q \int_{W_{D}}\left(\rho^{2} \nabla F \cdot \vec{e}_{1}\right)\left(z_{i}, x_{2}, \ldots, x_{d}\right) \mathrm{d}\left(x_{2}, \ldots, x_{d}\right),
\end{aligned}
$$

where $z_{1}=0, z_{2}=L_{1}$ and $\vec{e}_{1}=(1,0, \ldots, 0)^{T}$. We set

$$
I(F, \rho)=I_{1}(F, \rho) .
$$

In (2.7) we have to evaluate the gradient $\nabla F$ along $\Gamma_{D}$ which will not be possible for any $F$ in $H^{1}(W)$. This technical difficulty can easily be handled by using an alternative formula for $I(F, \rho)$ : 
- If $d=1$ then $W=] 0, L_{1}[$ and the current density $J=\vec{J}$ will be constant. Thus,

$$
I(F, \rho)=-\frac{q}{L_{1}} \int_{0}^{L_{1}} J \cdot 1 \mathrm{~d} \mathbf{x} \quad \text { for } d=1 .
$$

- For $d>1$ then we introduce for arbitrary $l \in] 0, L_{1}[$ the cuboid

$$
\left.W_{l}=\right] 0, l[\times] 0, L_{2}[\times \ldots \times] 0, L_{d}[\subset W .
$$

Recall that $\vec{J}$ is divergence-free and that there is no current-flow across the Neumann-boundary. Therefore, we obtain for $l \in] 0, L_{1}[$

$$
\begin{aligned}
0 & =\int_{W_{l}} \nabla \cdot \vec{J} \mathrm{~d} \mathbf{x}=\int_{\partial W_{l}} \vec{J} \cdot \nu \mathrm{d} \mathbf{s} \\
& =-\int_{\Gamma_{D}^{1}} \vec{J} \cdot \vec{e}_{1} \mathrm{~d} \mathbf{s}+\int_{\Gamma_{D}^{2}} \vec{J} \cdot \vec{e}_{1} \mathrm{~d} \mathbf{s}+\int_{\Gamma_{N} \cap \partial W_{l}} \vec{J} \cdot \nu \mathrm{d} \mathbf{s} \\
& =\int_{W_{D}}\left(\left(\vec{J} \cdot \vec{e}_{1}\right)\left(l, x_{2}, \ldots, x_{d}\right)-\left(\vec{J} \cdot \vec{e}_{1}\right)\left(0, x_{2}, \ldots, x_{d}\right)\right) \mathrm{d}\left(x_{2}, \ldots, x_{d}\right) .
\end{aligned}
$$

Consequently, we deduce from (2.7)

$$
\begin{aligned}
I(F, \rho) & =I_{1}(F, \rho)=(-1) q \int_{W_{D}}\left(\vec{J} \cdot \vec{e}_{1}\right)\left(0, x_{2}, \ldots, x_{d}\right) \mathrm{d}\left(x_{2}, \ldots, x_{d}\right) \\
& \left.=-q \int_{W_{D}}\left(\vec{J} \cdot \vec{e}_{1}\right)\left(l, x_{2}, \ldots, x_{d}\right) \mathrm{d}\left(x_{2}, \ldots, x_{d}\right) \quad \text { for } l \in\right] 0, L_{1}[,
\end{aligned}
$$

such that via integration from $l=0$ to $l=L_{1}$,

$$
I(F, \rho)=-\frac{q}{L_{1}} \int_{W} \vec{J} \cdot \vec{e}_{1} \mathrm{~d} \mathbf{x}=-\frac{q}{L_{1}} \int_{W} \rho^{2} \nabla F \cdot \vec{e}_{1} \mathrm{~d} \mathbf{x} \quad \text { for } d \in\{2,3\} .
$$

In the sequel we use formulae $(2.8)$ and $(2.9)$ as definitions of $I(F, \rho)$, see Section 3.

\section{The optimal control problem}

In this section we introduce the optimal control problem to determine amongst a class of admissible barrier functions the optimal $B$ to achieve a desired IV-curve. Further, we prove existence of optimal solutions.

We suppose the set of admissible barrier functions to be parametrized by $\ell, \ell \in \mathbb{N}^{*}$, variables. $U_{\text {ad }}$ is the set of admissible controls. We assume:

$$
\begin{aligned}
& \text { The set } U_{\mathrm{ad}}, \emptyset \neq U_{\mathrm{ad}} \subseteq \mathbb{R}^{\ell} \text { of control parameters is } \\
& \text { compact. The mapping } \\
& \qquad B: W \times U_{\mathrm{ad}} \rightarrow \mathbb{R} \\
& \text { is bounded and for all } x \in W \text { the partial function } \\
& B(\mathbf{x} ; \cdot) \text { is in } C^{(2,1)}\left(U_{\mathrm{ad}}\right) \text {. The set of all partial functions } \\
& \qquad \mathcal{B}_{\mathrm{ad}}=\left\{B\left(\cdot ; u_{1}, \ldots, u_{\ell}\right):\left(u_{1}, \ldots, u_{\ell}\right) \in U_{\mathrm{ad}}\right\} \\
& \text { is the set of admissible barrier functions. }
\end{aligned}
$$

The gradient $\nabla_{u} B(\cdot ; u)$ is a row vector with $\ell$ components. Let us present an example for the set $U_{\text {ad }}$ and $\mathcal{B}_{\text {ad }}$. 
EXAMPLE 3.1. Let $\left.\phi: \mathbb{R}^{d} \times\right] 0, \infty\left[\rightarrow\left[0, \infty\left[\right.\right.\right.$ be a function in $C_{c}^{\infty}\left(\mathbb{R}^{d} \times\right] 0,1 / 10[)$ such that for all $\epsilon \in] 0,1 / 10$ [ the function $\phi(\cdot, \epsilon)$ is supported in $]-1,1\left[\times \mathbb{R}^{d-1} . \phi\right.$ may be generated via mollifying a step function, where $\epsilon$ is a mollifying parameter. We obtain $\Phi:]-1,1\left[\times W_{D} \times\right] 0, \infty[\rightarrow \mathbb{R}$ by restricting $\phi$ to $]-1,1\left[\times W_{D} \times\right] 0, \infty[$. Then we choose $\left.\theta_{1}, \ldots, \theta_{m} \in\right] 0, L_{1}\left[\right.$ with $0<\theta_{1}<\ldots<\theta_{m}<L_{1}$ and we set $\delta:=\min \left\{\theta_{j}-\theta_{j-1}: 2 \leq j \leq m\right\}$ for $m \in \mathbb{N}$. Putting, e.g.,

$$
U_{\mathrm{ad}}=[a, A]^{m} \times[\delta / 10,9 \delta / 10]^{m} \times[1 / 100,1 / 20]
$$

for some $a, A \in \mathbb{R}$ with $0 \leq a<A$ and defining $B: W \times U_{\text {ad }} \rightarrow \mathbb{R}$ by

$$
B\left(x ; \alpha_{1}, \ldots, \alpha_{m}, \xi_{1}, \ldots, \xi_{m}, \epsilon\right)=\sum_{j=1}^{m} \alpha_{j} \Phi\left(\frac{x_{1}-\theta_{j}}{\xi_{j}}, x_{2}, \ldots, x_{d}, \epsilon\right),
$$

we obtain a function $B$ meeting the requirements of $(\mathbf{A} 7)$ with $\ell=1+2 m$. Obviously each $B$ is the sum of re-scaled, shifted, compactly supported test functions whose supports do not intersect.

For control parameters $u \in U_{\text {ad }}$ the quantum barrier function is $B(\cdot ; u)$ and the state variables $F, \rho$, and $V$ are $H^{1}$ solutions of the scaled QDD model (2.3) which has, according to Theorem 2.4, for given $B(\cdot ; u)$ at least one $H^{1}$ solution.

We proceed by introducing the cost functional $J: H_{b} \times H_{b} \times \mathbb{R}^{\ell} \rightarrow[0, \infty)$ by

$$
J(F, \rho, u)=\frac{1}{2}\left|I(F, \rho)-I_{d}\right|^{2}+\sum_{i=1}^{\ell} \frac{\beta_{i}}{2}\left|u_{i}\right|^{2},
$$

where the current functional is

$$
I(F, \rho)=-\frac{q}{L_{1}} \int_{W} \rho^{2} \nabla F \cdot \vec{e}_{1} \mathrm{~d} \mathbf{x},
$$

$I_{d} \in \mathbb{R}$ is a given desired current, and the $\beta_{i}$ 's are positive regularization scalars.

LEMma 3.2. The current functional $I: H_{b} \times H_{b} \rightarrow \mathbb{R}$ is twice continuously Fréchetdifferentiable and its second Fréchet derivative is Lipschitz-continuous.

A proof of Lemma 3.2 is given in the appendix.

Lemma 3.3. The cost functional $J$ is weakly (lower semi-) continuous. Moreover, $J$ is twice continuously Fréchet-differentiable and its second Fréchet derivative is Lipschitzcontinuous.

Proof. First we show that the cost $J$ is weakly continuous. For this purpose let $\left\{\left(F^{n}, \rho^{n}, u^{n}\right)\right\}_{n \in \mathbb{N}}$ be a sequence in $H_{b} \times H_{b} \times \mathbb{R}^{\ell}$ with

$$
\left(F^{n}, \rho^{n}, u^{n}\right) \rightarrow\left(F^{*}, \rho^{*}, u^{*}\right) \text { in } H_{b} \times H_{b} \times \mathbb{R}^{\ell} \text { as } n \rightarrow \infty .
$$

Since $H^{1}(W)$ is compactly embedded in $L^{4}(W)$ for $d \leq 3$ (see [1]), we have

$$
\left(\rho^{n}\right)^{2} \rightarrow\left(\rho^{*}\right)^{2} \text { in } L^{2}(W) \text { as } n \rightarrow \infty .
$$

Moreover, $\nabla F^{n} \rightarrow \nabla F^{*}$ in $L^{2}\left(W ; \mathbb{R}^{d}\right)$ as $n \rightarrow \infty$ which implies

$$
\lim _{n \rightarrow \infty}-\frac{q}{L_{1}} \int_{W}\left(\left(\rho^{n}\right)^{2} \nabla F^{n}-\left(\rho^{*}\right)^{2} \nabla F^{*}\right) \cdot \vec{e}_{1} \mathrm{~d} \mathbf{x}=0 .
$$


Consequently,

$$
\lim _{n \rightarrow \infty}\left|I\left(F^{n}, \rho^{n}\right)-I_{d}\right|^{2}=\left|I\left(F^{*}, \rho^{*}\right)-I_{d}\right|^{2}
$$

Since $u^{n} \rightarrow u^{*}$ in $\mathbb{R}^{\ell}$ we directly infer that

$$
\lim _{n \rightarrow \infty}\left|u_{i}^{n}\right|^{2}=\left|u_{i}^{*}\right| \quad \text { for } 1 \leq i \leq \ell .
$$

From (3.1) and (3.2) it follows that $J$ is weakly continuous and consequently weakly lower semi-continuous as well.

Due to Lemma 3.2 the operator $I$ is twice continuously Fréchet-differentiable. Consequently, $J$ is twice continuously Fréchet-differentiable at any $(\bar{F}, \bar{\rho}, \bar{u}) \in H_{b} \times H_{b} \times \mathbb{R}^{\ell}$. For directions $(F, \rho, u),(\tilde{F}, \tilde{\rho}, \tilde{u}) \in H_{b} \times H_{b} \times \mathbb{R}^{\ell}$ the first and second derivatives are

$$
\nabla J(\bar{F}, \bar{\rho}, \bar{u})(F, \rho, u)=\left(I(\bar{F}, \bar{\rho})-I_{d}\right) \nabla I(\bar{F}, \bar{\rho})(F, \rho)+\sum_{i=1}^{\ell} \beta_{i} \bar{u}_{i} u_{i}
$$

and

$$
\begin{aligned}
& \nabla^{2} J(\bar{F}, \bar{\rho}, \bar{u})((\tilde{F}, \tilde{\rho}, \tilde{u}),(F, \rho, u)) \\
&= \nabla I(\bar{F}, \bar{\rho})(F, \rho) \nabla I(\bar{F}, \bar{\rho})(\tilde{F}, \tilde{\rho})+\sum_{i=1}^{\ell} \beta_{i} \tilde{u}_{i} u_{i} \\
&+\left(I(\bar{F}, \bar{\rho})-I_{d}\right) \nabla^{2} I(\bar{F}, \bar{\rho})((\tilde{F}, \tilde{\rho}),(F, \rho))
\end{aligned}
$$

where $\nabla I$ and $\nabla^{2} I$ are given by formulas (A.8) and (A.9) in the appendix, respectively. Since the mapping $(\bar{F}, \bar{\rho}) \mapsto \nabla^{2} I(\bar{F}, \bar{\rho})$ is Lipschitz-continuous, we conclude that $(\bar{F}, \bar{\rho}, \bar{u}) \mapsto \nabla^{2} J(\bar{F}, \bar{\rho}, \bar{u})$ is Lipschitz-continuous as well.

REMARK 3.4. Advanced numerical optimization methods - like sequential quadratic programming (SQP) techniques - are known to have second-order rate of convergence properties provided the cost functional and the constraints are twice continuously Fréchet-differentiable with at least locally Lipschitz-continuous second derivatives. Due to Lemma 3.3 the cost functional has these smoothness properties. The smoothness properties of the constraints will be addressed in Lemma 4.1.

To deal with the QDD model in an abstract form, let us define the closed subset $X_{\text {ad }}$ of the Banach space $X=\left(H_{b}\right)^{3} \times \mathbb{R}^{\ell}$ and the Hilbert space $Y$ as follows

$$
X_{\mathrm{ad}}=\left(H_{b}\right)^{3} \times U_{\mathrm{ad}} \quad Y=\left(H_{\circ}\right)^{3} \times\left(H^{1 / 2}\left(\Gamma_{D}\right)^{\prime}\right)^{3},
$$

endowed with their natural product topology. Recall that $H_{\circ}=H_{0}^{1}\left(W \cup \Gamma_{N}\right)$. We identify the dual $Y^{\prime}$ of $Y$ with

$$
\left(H_{\circ}^{\prime}\right)^{3} \times\left(H^{1 / 2}\left(\Gamma_{D}\right)\right)^{3}
$$

Next we introduce the non-linear operator

$$
e=\left(e^{1}, e^{2}, e^{3}, e^{4}, e^{5}, e^{6}\right): X \rightarrow Y^{\prime}
$$


via

$$
\begin{aligned}
\left\langle e^{1}(x), \varphi\right\rangle_{H_{\circ}^{\prime}, H_{\circ}} & =\int_{\Omega} \rho^{2} \nabla F \cdot \nabla \varphi \mathrm{d} \mathbf{x}, \\
\left\langle e^{2}(x), \varphi\right\rangle_{H_{\circ}^{\prime}, H_{\circ}} & =\int_{W} \varepsilon^{2} \nabla \rho \cdot \nabla \varphi+\rho\left(V+B(\cdot ; u)+\log \left(\rho^{2}\right)-F\right) \varphi \mathrm{d} \mathbf{x}, \\
\left\langle e^{3}(x), \varphi\right\rangle_{H_{\circ}^{\prime}, H_{\circ}} & =\int_{W} \lambda^{2} \nabla V \cdot \nabla \varphi+\left(C-\rho^{2}\right) \varphi \mathrm{d} \mathbf{x}
\end{aligned}
$$

for $\varphi \in H_{\circ}$ and

$$
e^{4}(x)=\tau_{D}\left(F-F_{D}\right), \quad e^{5}(x)=\tau_{D}\left(\rho-\rho_{D}\right), \quad e^{6}(x)=\tau_{D}\left(V-V_{D}\right),
$$

where $x=(F, \rho, V, u) \in X, B=B(\cdot ; u)$ and

$$
\tau_{D}: H^{1}(\Omega) \rightarrow H^{1 / 2}\left(\Gamma_{D}\right)
$$

is the canonical (continuous, surjective) projection from $H^{1}(\Omega)$ onto $H^{1 / 2}\left(\Gamma_{D}\right)$.

REMARK 3.5. One can also work on the affine space $x_{D}+X$ with the element $x_{D}=$ $\left(F_{D}, \varrho_{D}, V_{D}, 0\right) \in X$ and avoid the introduction of the constraints $e^{i}, i=4,5,6$ without getting any different results.

The optimal control problem can be expressed in an abstract form as

$$
\min J(F, \rho, u) \quad \text { s.t. } \quad x=(F, \rho, V, u) \in X_{\text {ad }} \text { and } e(x)=0 \text { in } Y^{\prime} \text {. }
$$

Theorem 3.6. Suppose (A1)-(A7). Then $(\mathbf{P})$ has at least one global optimal solution $x^{*}=\left(F^{*}, \rho^{*}, V^{*}, u^{*}\right) \in X_{\mathrm{ad}}$.

Proof. By $(\mathbf{A} 7)$ we have $U_{\text {ad }} \neq \emptyset$. Due to Theorem 2.4, the set

$$
E=\left\{x=(F, \rho, V, u) \in X \mid e(x)=0 \text { in } Y^{\prime}\right\}
$$

is non-empty. Due to $(\mathbf{A} 7)$, there is a constant $c_{1}>0$ with $\|B(\cdot ; u)\|_{L^{\infty}(W)} \leq c_{1}$ for all $u \in U_{\text {ad }}$.

Suppose that for chosen $u \in U_{\text {ad }}$ the triple $(F, \rho, V) \in H_{b}^{3}$ is a $H^{1}$ solution of (2.3) with $B=B(\cdot ; u)$. Then we infer from $(2.6)$ that $F, \rho$, and $V$ are bounded in $H_{b}$ and there exists a $\zeta \geq 0$ with

$$
\zeta=\inf \left\{J(F, \rho, u) \mid(F, \rho, V, u) \in X_{\text {ad }} \text { satisfies (2.3) weakly }\right\} .
$$

Let us consider a minimizing sequence $\left\{x^{n}\right\}_{n \in \mathbb{N}}, x^{n}=\left(F^{n}, \rho^{n}, V^{n}, u^{n}\right)$, in $X_{\text {ad }}$, i.e., $\zeta=\lim _{n \rightarrow \infty} J\left(F^{n}, \rho^{n}, u^{n}\right)$ holds. We define the sequence $\left\{B^{n}\right\}_{n \in \mathbb{N}}$ via $B^{n}=B\left(\cdot ; u_{n}\right)$, $n \in \mathbb{N}$. Then $x^{n}$ satisfies (2.3) for all $n \in \mathbb{N}$. Since $U_{\text {ad }}$ is a compact subset of $\mathbb{R}^{\ell}$, there is $u^{*} \in U_{\text {ad }}$ such that, eventually by passing to a subsequence but without changing notations, $u^{n} \rightarrow u^{*}$ in $\mathbb{R}^{\ell}$. This implies that

$$
B^{n} \rightarrow B^{*}=B\left(\cdot ; u^{*}\right) \quad \text { as } n \text { tends to } \infty
$$

Moreover, we find from (2.6) that that

$$
\left(F^{n}, \rho^{n}, V^{n}\right) \rightarrow\left(F^{*}, \rho^{*}, V^{*}\right) \text { in } H^{1}(W)^{3} \text { as } n \rightarrow \infty
$$


and $\left\|F^{n}\right\|_{L^{\infty}(W)}+\left\|\rho^{n}\right\|_{L^{\infty}(W)}+\left\|V^{n}\right\|_{L^{\infty}(W)}$ is bounded, see Theorem 2.4. Let $x^{*}=$ $\left(F^{*}, \rho^{*}, V^{*}, u^{*}\right)$. Next we prove that $\left(F^{*}, \rho^{*}, V^{*}\right)$ solves $(2.3)$ weakly. Since $H^{1}(W)$ is compactly embedded into $L^{4}(W)$ for $d \leq 3$, we infer

$$
\rho^{n} \rightarrow \rho^{*} \text { in } L^{4}(W) \text { as } n \rightarrow \infty,
$$

which implies that

$$
\left(\rho^{n}\right)^{2} \rightarrow\left(\rho^{*}\right)^{2} \text { in } L^{2}(W) \text { as } n \rightarrow \infty .
$$

From $\nabla F^{n} \rightarrow \nabla F^{*}$ in $L^{2}\left(W ; \mathbb{R}^{d}\right)$ as $n$ tends to $\infty$ and from (3.8) we infer

$$
\lim _{n \rightarrow \infty} \int_{W}\left(\rho^{n}\right)^{2} \nabla F^{n} \cdot \nabla \varphi \mathrm{d} \mathbf{x}=\int_{W}\left(\rho^{*}\right)^{2} \nabla F^{*} \cdot \nabla \varphi \mathrm{d} \mathbf{x} \quad \text { for all } \varphi \in H_{\circ},
$$

i.e., $e^{1}\left(x^{*}\right)=0$ in $H_{\circ}^{\prime}$. Utilizing (3.7) we deduce via the continuity of the function $\phi:[0,+\infty[\rightarrow \mathbb{R}, \phi(t)=t \log t$ for $t>0$ and $\phi(0)=0$ that

$$
\lim _{n \rightarrow \infty} \log \left(\rho^{n}(\mathbf{x})^{2}\right)=\log \left(\rho^{*}(\mathbf{x})^{1}\right) \quad \text { for } \quad \mathbf{x} \in W \text { a.e. }
$$

Furthermore, $\left\|\rho^{n}\right\|_{L^{\infty}(W)}$ is bounded. Hence, there exists a constant $c_{2}>0$ such that

$$
\left\|2 \rho^{n} \log \rho^{n}\right\|_{L^{\infty}(W)} \leq c_{2} .
$$

Applying Lebesgue's dominated convergence theorem (see, e.g. [14]) we obtain

$$
\lim _{n \rightarrow \infty} \int_{W} 2 \rho^{n} \log \left(\rho^{n}\right) \varphi \mathrm{d} \mathbf{x}=\int_{W} 2 \rho^{*} \log \left(\rho^{*}\right) \varphi \mathrm{d} \mathbf{x} \quad \text { for all } \varphi \in H_{\circ} .
$$

From (3.6) and (3.7) we conclude for all $\varphi \in H_{\circ}$,

$$
\lim _{n \rightarrow \infty}\left\langle e^{2}\left(x^{n}\right), \varphi\right\rangle_{H_{\circ}^{\prime}, H_{\circ}}=\left\langle e^{2}\left(x^{*}\right), \varphi\right\rangle_{H_{\circ}^{\prime}, H_{\circ}} .
$$

Again applying (3.6) and (3.7) we find that, for all $\varphi \in H_{\circ}$,

$$
\lim _{n \rightarrow \infty}\left\langle e^{3}\left(x^{n}\right), \varphi\right\rangle_{H_{\circ}^{\prime}, H_{\circ}}=\left\langle e^{3}\left(x^{*}\right), \varphi\right\rangle_{H_{\circ}^{\prime}, H_{\circ}} .
$$

Now we turn to the constraint $e^{4}\left(x^{n}\right)=0$. By assumption, $F_{D} \in H_{b}$. Hence,

$$
F^{n}-F_{D} \rightarrow F^{*}-F_{D} \text { in } H_{\circ} \text { as } n \rightarrow \infty \text {. }
$$

Moreover, $F^{n}-F_{D} \in H_{\circ}$ for all $n \in \mathbb{N}$. Thus, $F^{*}-F_{D} \in H_{\circ}$ and

$$
e^{4}\left(x^{*}\right)=\tau\left(F^{*}-F_{D}\right)=0 .
$$

Analogously, we obtain

$$
e^{5}\left(x^{*}\right)=e^{6}\left(x^{*}\right)=0 .
$$

Summarizing, (3.9), (3.11)-(3.14) imply $e\left(x^{*}\right)=0$. By Lemma 3.3 the cost functional is weakly continuous. 


\section{First-order necessary optimality conditions}

This section is devoted to study first-order necessary optimality conditions for $(\mathbf{P})$. If a constrained qualification holds, there exist Lagrange multipliers satisfying a coupled dual system of three elliptic equations.

Problem $(\mathbf{P})$ is a non-convex programming problem so that different local minima may occur. Typically a numerical method will approximates a local minimum close to its starting value. Hence, we do not restrict our investigations to global solutions of $(\mathbf{P})$. We assume a fixed reference solution $x^{*}=\left(F^{*}, \rho^{*}, V^{*}, u^{*}\right)$ and investigate certain first-order optimality conditions. For that purpose we introduce the Lagrange functional $\mathcal{L}: X \times Y \rightarrow \mathbb{R}$ associated with $(\mathbf{P})$ :

$$
\begin{aligned}
& \mathcal{L}(x, p)=J(F, \rho, u)+\langle e(x), p\rangle_{Y^{\prime}, Y} \\
& =\frac{1}{2}\left|I(F, \rho)-I_{d}\right|^{2}+\sum_{i=1}^{\ell} \frac{\beta_{i}}{2}\left|u_{i}\right|^{2}+\int_{\Omega} \rho^{2} \nabla F \cdot \nabla p_{F} \mathrm{~d} \mathbf{x} \\
& \quad+\int_{W} \varepsilon^{2} \nabla \rho \cdot \nabla p_{\rho}+\rho\left(V+B(\cdot ; u)+\log \left(\rho^{2}\right)-F\right) p_{\rho} \mathrm{d} \mathbf{x}, \\
& \quad+\int_{W} \lambda^{2} \nabla V \cdot \nabla p_{V}+\left(C-\rho^{2}\right) p_{V} \mathrm{~d} \mathbf{x}+\left\langle\tau_{D}\left(F-F_{D}\right), p_{F_{D}}\right\rangle_{H^{1 / 2}\left(\Gamma_{D}\right)^{\prime}, H^{1 / 2}\left(\Gamma_{D}\right)} \\
& \quad+\left\langle\tau_{D}\left(\rho-\rho_{D}\right), p_{\rho_{D}}\right\rangle_{H^{1 / 2}\left(\Gamma_{D}\right)^{\prime}, H^{1 / 2}\left(\Gamma_{D}\right)}+\left\langle\tau_{D}\left(V-V_{D}\right), p_{V_{D}}\right\rangle_{H^{1 / 2}\left(\Gamma_{D}\right)^{\prime}, H^{1 / 2}\left(\Gamma_{D}\right)},
\end{aligned}
$$

where $x=(F, \rho, V, u) \in X$ and $p=\left(p_{F}, p_{\rho}, p_{V}, p_{F_{D}}, p_{\rho_{D}}, p_{V_{D}}\right) \in Y$.

Due to Lemma 3.3 the cost functional $J$ is continuously Fréchet differentiable and its second derivative is Lipschitz-continuous in $X$. In the next lemma we state that the operator $e$ is twice continuously Fréchet-differentiable. A proof is given in the appendix.

Lemma 4.1. For every $\bar{x}=(\bar{F}, \bar{\rho}, \bar{V}, \bar{u}) \in X$ with $\operatorname{essinf}_{W} \bar{\rho}>0$ the mapping $\bar{x} \rightarrow e(\bar{x}) \in$ $Y^{\prime}$ is twice continuously Fréchet-differentiable and its second Fréchet-derivatiuve is locally Lipschitz-continuous.

To formulate first-order necessary optimality conditions we have to ensure that the solution $x^{*}$ to $(\mathbf{P})$ is a regular point.

Definition 4.2. Let $\bar{x}=(\bar{F}, \bar{\rho}, \bar{V}, \bar{u})$ be a feasible point for $(\mathbf{P})$, i.e.,

$$
\bar{x} \in \mathcal{F}(\mathbf{P})=\left\{\bar{x} \in X \mid e(\bar{x})=0 \text { in } Y^{\prime} \text { and } \bar{x} \in X_{\mathrm{ad}}\right\}
$$

Then, $\bar{x}$ is called a regular point if the linearization $\nabla e(\bar{x}): X \rightarrow Y^{\prime}$ of the operator $e$ at $\bar{x}$ is surjective.

Due to Lemma 4.1 the linear operator $\nabla e(\bar{x})$ is bounded provided $\operatorname{essinf}_{W} \bar{\rho}>0$ is satisfied. Certainly, $\nabla e\left(x^{*}\right)$ will be surjective if for any given element $f=$ $\left(f_{F}, f_{\rho}, f_{V}, f_{F_{D}}, f_{\rho_{D}}, f_{V_{D}}\right) \in Y^{\prime}$ there exists a solution $x=(F, \rho, V, u) \in X$ of

$$
\nabla e\left(x^{*}\right) x=f \quad \text { in } Y^{\prime}
$$


The element $x \in X$ is a solution of (4.1) if and only if

$$
\begin{aligned}
& \int_{W}\left(\left(\rho^{*}\right)^{2} \nabla F+2 \rho^{*} \rho \nabla F^{*}\right) \cdot \nabla \varphi \mathrm{d} \mathbf{x}=\left\langle f_{D}, \varphi\right\rangle_{H_{\circ}^{\prime}, H_{\circ}} \\
& \int_{W} \varepsilon^{2} \nabla \rho \cdot \nabla \varphi+\rho\left(V^{*}+B^{*}+\log \left(\left(\rho^{*}\right)^{2}\right)-F^{*}\right) \varphi \mathrm{d} \mathbf{x} \\
& \quad+\int_{W}\left(\rho^{*}\left(V+\nabla_{u} B^{*} u-F\right)+2 \rho\right) \varphi \mathrm{d} \mathbf{x}=\left\langle f_{\rho}, \varphi\right\rangle_{H_{\circ}^{\prime}, H_{\circ}}, \\
& \int_{W} \lambda^{2} \nabla V \cdot \nabla \varphi+2 \rho^{*} \rho \varphi \mathrm{d} \mathbf{x}=\left\langle f_{V}, \varphi\right\rangle_{H_{\circ}^{\prime}, H_{\circ}}
\end{aligned}
$$

for all $\varphi \in H_{\circ}$ and

$$
F=f_{F_{D}}, \quad \rho=f_{\rho_{D}}, \quad V=f_{V_{D}} \quad \text { in } H^{1 / 2}\left(\Gamma_{D}\right),
$$

where $B^{*}=B\left(\cdot ; u^{*}\right)$ and $\nabla_{u} B^{*}=\nabla_{u} B\left(\cdot ; u^{*}\right)^{T}$. System $(4.2)$ is the variational formulation of the coupled linear elliptic system

$$
\begin{array}{rlrl}
-\nabla \cdot\left(\left(\rho^{*}\right)^{2} \nabla F+2 \rho^{*} \nabla F^{*} \rho\right) & =f_{D} & & \text { in } W, \\
-\varepsilon^{2} \Delta \rho+\rho\left(V^{*}+B^{*}+\log \left(\left(\rho^{*}\right)^{2}\right)-F^{*}+2\right) & & \\
+\rho^{*}\left(V+\nabla_{u} B^{*} u-F\right) & =f_{\rho} & & \text { in } W, \\
-\lambda^{2} \Delta V+2 \rho^{*} \rho & =f_{V} & & \text { in } W, \\
F & =f_{F_{D}} & & \text { on } \Gamma_{D}, \\
\rho & =f_{\rho_{D}} & & \text { on } \Gamma_{D}, \\
V & =f_{V_{D}} & & \text { on } \Gamma_{D}, \\
\frac{\partial F}{\partial \nu}=\frac{\partial \rho}{\partial \nu}=\frac{\partial V}{\partial \nu} & =0 & & \text { on } \Gamma_{N} .
\end{array}
$$

Due to the lack of coercitivity it is far from obvious that, settled on grounds of the Fredholm alternative, (4.3) has a weak solution. On the other hand it is seemingly also quite difficult to verify that (4.3) has no solution: The universe of linear (operator) equations is certainly much larger than the set of linear (operator) equations for which the solvability question can be settled just in terms of the involved parameters. Comparing the situation with the finite-dimensional case (aside from the use of determinants which provide to the authors' knowledge no tractable concept extendable to infinite-dimensional settings): Unless rather specific information about the coefficient matrix of a linear system (positivity, self-adjointness whatsoever) are available one will not be able to say anything about the set of solutions - except, of course, that the probability that a $n$ times $n$ linear system with, let us say, somewhere uniformly distributed coefficients is uniquely solvable is one. So from which properties of the coefficients could we deduce unique solvability in case of "unstructuredness"? Even in the finite-dimensional case the authors have no answer to that question and we do not think things become easier in infinite dimensional settings. As a conclusion, to decide whether $\nabla e\left(x^{*}\right)$ is surjective or not is not in the authors' scope. Possibly, further numerical investigations may allow to gain more insight in the subject.

On the other hand, for practical purposes the existence of associated Lagrange multipliers is quite useful to obtain information on the local minimizer $x^{*}$. Since we are interested in these information let us assume

$$
\nabla e\left(x^{*}\right): X \rightarrow Y^{\prime} \text { is surjective. }
$$


REMARK 4.3. If $\nabla e\left(x^{*}\right)$ is not surjective from $X \rightarrow Y^{\prime}$ but its extension on a larger space $\tilde{X}, X$ densely embedded into $\tilde{X}$, possesses specific properties (weakly singular optimal control problem [9]) one can also prove existence of Lagrange multipliers.

If (A8) is satisfied, the point $x^{*}$ is a regular point. The KKT theorem [10, Theorem 3.2] implies that there exists a unique associated Lagrange multiplier $p^{*}=$ $\left(p_{F}^{*}, p_{\rho}^{*}, p_{V}^{*}, p_{F_{D}}^{*}, p_{\rho_{D}}^{*}, p_{V_{D}}^{*}\right) \in Y$ satisfying

$$
\nabla_{(F, \rho, V)} e\left(x^{*}\right)^{\star} p^{*}=-\nabla_{(F, \rho)} J\left(F^{*}, \rho^{*}, u^{*}\right) \quad \text { in }\left(H_{b}^{\prime}\right)^{3},
$$

where $\nabla_{(F, \rho, V)} e\left(x^{*}\right)^{\star}: Y \rightarrow\left(H_{b}^{\prime}\right)^{3}$ is the dual operator of $\nabla_{(F, \rho, V)} e\left(x^{*}\right)$, i.e.,

$$
\left\langle\nabla_{(F, \rho, V)} e\left(x^{*}\right)^{\star} p,(F, \rho, V)\right\rangle_{\left(H_{b}^{\prime}\right)^{3}, H_{b}}=\left\langle\nabla_{(F, \rho, V)} e\left(x^{*}\right)(F, \rho, V), p\right\rangle_{Y^{\prime}, Y}
$$

for all $(F, \rho, V, p) \in\left(H_{b}\right)^{3} \times Y$. Certainly, (4.4) and the first-order necessary optimality condition

$$
\nabla_{(F, \rho, V)} \mathcal{L}\left(x^{*}, p^{*}\right)=0 \quad \text { in }\left(H_{b}^{\prime}\right)^{3}
$$

are equivalent.

In the following proposition we state that the Lagrange multipliers satisfy a coupled linear elliptic system.

Proposition 4.4. Let (A8) hold. The Lagrange multipliers $p_{F}^{*}, p_{\rho}^{*}$ and $p_{V}^{*}$ satisfy the following coupled linear system

$$
\begin{aligned}
-\nabla\left(\left(\rho^{*}\right)^{2} \nabla p_{F}^{*}\right)-\rho^{*} p_{\rho}^{*} & =-\left(I\left(F^{*}, \rho^{*}\right)-I_{d}\right) \nabla_{F} I\left(F^{*}, \rho^{*}\right) \\
-\varepsilon \Delta p_{\rho}^{*}+2 \rho^{*} \nabla F^{*} \cdot \nabla p_{F}^{*}-2 \rho^{*} p_{V}^{*} & \\
+\left(V^{*}+B^{*}+\log \left(\left(\rho^{*}\right)^{2}\right)-F^{*}+2\right) p_{\rho}^{*} & =-\left(I\left(F^{*}, \rho^{*}\right)-I_{d}\right) \nabla_{\rho} I\left(F^{*}, \rho^{*}\right) \\
-\lambda^{2} \Delta p_{V}^{*}+\rho^{*} p_{\rho}^{*} & =0
\end{aligned}
$$

weakly in $H_{\circ}$, together with homogeneous Neumann boundary conditions on $\Gamma_{N}$. Moreover,

$$
p_{F_{D}}^{*}=\left(\rho^{*}\right)^{2} \frac{\partial p_{F}^{*}}{\partial \nu}, \quad p_{\rho_{D}}=\varepsilon \frac{\partial p_{\rho}^{*}}{\partial \nu}, \quad p_{V_{D}}^{*}=\lambda^{2} \frac{\partial p_{\rho}^{*}}{\partial \nu} \quad \text { in } H^{1 / 2}\left(\Gamma_{D}\right)^{\prime} .
$$

Proof. Since $\left(p_{F}^{*}, p_{\rho}^{*}, p_{V}^{*}\right) \in\left(H_{\circ}\right)^{3}$ the functions $p_{F}^{*}, p_{\rho}^{*}$ and $p_{V}^{*}$ satisfy homogeneous Dirichlet boundary conditions on $\Gamma_{D}$. We choose an arbitrary $(F, \rho, V) \in\left(H_{b}\right)^{3}$. Recall that the gradient $\nabla_{u} B\left(\cdot ; u^{*}\right)$ is a row vector. As outlined in the proof of Lemma 4.1, see appendix, we have

$$
\begin{aligned}
& \left\langle\nabla_{(F, \rho, V)} e\left(x^{*}\right)^{*} p^{*},(F, \rho, V)\right\rangle_{\left(H_{b}^{\prime}\right)^{3},\left(H_{b}\right)^{3}}=\left\langle\nabla_{(F, \rho, V)} e\left(x^{*}\right) x, p^{*}\right\rangle_{Y^{\prime}, Y} \\
= & \int_{W}\left(2 \rho^{*} \rho \nabla F^{*}+\left(\rho^{*}\right)^{2} \nabla F\right) \cdot \nabla p_{F}^{*} \mathrm{~d} \mathbf{x} \\
& +\int_{W} \rho\left(V^{*}+B\left(\cdot ; u^{*}\right)+\log \left(\left(\rho^{*}\right)^{2}\right)-F^{*}+2\right) p_{\rho}^{*} \mathrm{~d} \mathbf{x} \\
& +\int_{W} \varepsilon \nabla p_{\rho}^{*} \cdot \nabla \rho+\rho^{*}\left(V+\nabla_{u} B\left(\cdot ; u^{*}\right) u-F\right) p_{\rho}^{*} \mathrm{~d} \mathbf{x}
\end{aligned}
$$




$$
\begin{aligned}
& +\int_{W} \lambda^{2} \nabla p_{V}^{*} \cdot \nabla V-2 \rho^{*} p_{V}^{*} \rho \mathrm{d} \mathbf{x} \\
& +\left\langle p_{F_{D}}^{*}, F\right\rangle_{H^{1 / 2}\left(\Gamma_{D}\right)^{\prime}, H^{1 / 2}\left(\Gamma_{D}\right)}+\left\langle p_{\rho_{D}}^{*}, \rho\right\rangle_{H^{1 / 2}\left(\Gamma_{D}\right)^{\prime}, H^{1 / 2}\left(\Gamma_{D}\right)} \\
& +\left\langle p_{V_{D}}^{*}, V\right\rangle_{H^{1 / 2}\left(\Gamma_{D}\right)^{\prime}, H^{1 / 2}\left(\Gamma_{D}\right)} .
\end{aligned}
$$

Choosing $F \in H_{0}^{1}(W)$ and $\rho=0, V=0, u=0$ we derive from (4.6), (4.4) and (3.3)

$$
\begin{aligned}
& \left\langle\left(I_{d}-I\left(F^{*}, \rho^{*}\right)\right) \nabla_{F} I\left(F^{*}, \rho^{*}\right), F\right\rangle_{H_{b}^{\prime}, H_{b}} \\
= & \int_{W}\left(\rho^{*}\right)^{2} \nabla p_{F}^{*} \cdot \nabla F-\rho^{*} p_{\rho}^{*} F \mathrm{~d} \mathbf{x}=\left\langle-\nabla \cdot\left(\left(\rho^{*}\right)^{2} \nabla p_{F}^{*}\right)-\rho^{*} p_{\rho}^{*}, F\right\rangle_{H_{b}^{\prime}, H_{b}},
\end{aligned}
$$

which gives (4.5a) in $H^{-1}(W)$. Since $H_{\circ}$ is dense in $H_{0}^{1}(W),(4.7)$ holds in $H_{\circ}^{\prime}$. From (4.4), (4.6), and (4.7) we infer that

$$
\begin{aligned}
0= & \left\langle\left(I_{d}-I\left(F^{*}, \rho^{*}\right)\right) \nabla_{F} I\left(F^{*}, \rho^{*}\right), F\right\rangle_{H_{b}^{\prime}, H_{b}}-\int_{W}\left(\rho^{*}\right)^{2} \nabla p_{F}^{*} \cdot \nabla F-\rho^{*} p_{\rho}^{*} F \mathrm{~d} \mathbf{x} \\
& +\left\langle p_{F_{D}}^{*}, F\right\rangle_{H^{1 / 2}\left(\Gamma_{D}\right)^{\prime}, H^{1 / 2}\left(\Gamma_{D}\right)} \\
= & -\int_{\Gamma}\left(\rho^{*}\right)^{2} \frac{\partial p_{F}^{*}}{\partial \nu} F \mathrm{~d} \mathbf{s}+\left\langle p_{F_{D}}^{*}, F\right\rangle_{H^{1 / 2}\left(\Gamma_{D}\right)^{\prime}, H^{1 / 2}\left(\Gamma_{D}\right)}
\end{aligned}
$$

for all $F \in H_{b}$. If we choose $F \in H_{\circ}$, we find that $p_{F}^{*}$ satisfies homogeneous Neumann boundary conditions on $\Gamma_{N}$. On the other hand, if $F \in H_{0}^{1}\left(W \cup \Gamma_{D}\right)$ we obtain

$$
p_{F_{D}}^{*}=\left(\rho^{*}\right)^{2} \frac{\partial p_{F}^{*}}{\partial \nu} \quad \text { in } H^{1 / 2}\left(\Gamma_{D}\right)^{\prime} .
$$

Next we choose $\rho \in H_{0}^{1}(W)$ and $F=0, V=0, u=0$ we derive from (4.6), (4.4) and (3.3)

$$
\begin{aligned}
& \left\langle\left(I_{d}-I\left(F^{*}, \rho^{*}\right)\right) \nabla_{\rho} I\left(F^{*}, \rho^{*}\right), \rho\right\rangle_{H_{b}^{\prime}, H_{b}} \\
= & \int_{W} \varepsilon \nabla p_{\rho}^{*} \cdot \nabla \rho+2 \rho^{*} \nabla F^{*} \cdot \nabla p_{F}^{*} \rho+2 p_{\rho}^{*} \rho \mathrm{d} \mathbf{x} \\
& +\int_{W}\left(\left(V^{*}+B\left(\cdot ; u^{*}\right)+\log \left(\left(\rho^{*}\right)^{2}\right)-F^{*}\right) p_{\rho}^{*}-2 \rho^{*} p_{V}^{*}\right) \rho \mathrm{d} \mathbf{x} \\
= & \left\langle-\varepsilon \Delta p_{\rho}^{*}+2 \rho^{*} \nabla F^{*} \cdot \nabla p_{F}^{*}, \rho\right\rangle_{H_{b}^{\prime}, H_{b}} \\
& +\left\langle\left(V^{*}+B\left(\cdot ; u^{*}\right)+\log \left(\left(\rho^{*}\right)^{2}\right)-F^{*}+2\right) p_{\rho}^{*}-2 \rho^{*} p_{V}^{*}, \rho\right\rangle_{H_{b}^{\prime}, H_{b}},
\end{aligned}
$$

which gives $(4.5 \mathrm{~b})$ in $H_{\circ}^{\prime}$. Proceeding as above we find that $p_{\rho}^{*}$ satisfies homogeneous Neumann boundary conditions on $\Gamma_{N}$ and

$$
p_{\rho_{D}}^{*}=\varepsilon \frac{\partial p_{\rho}^{*}}{\partial \nu} \quad \text { in } H^{1 / 2}\left(\Gamma_{D}\right) .
$$

Choosing $V \in H_{0}^{1}(W)$ and $F=0, \rho=0, u=0$ we derive from (4.6), (4.4) and (3.3)

$$
0=\int_{W} \lambda^{2} \nabla p_{V}^{*} \cdot \nabla V+\rho^{*} p_{\rho}^{*} V \mathrm{~d} \mathbf{x}=\left\langle-\lambda^{2} \Delta p_{V}^{*}+\rho^{*} p_{\rho}^{*}, V\right\rangle_{H_{b}^{\prime}, H_{b}},
$$


which gives $(4.5 \mathrm{c})$ in $H_{\circ}^{\prime}$. As above, it follows that $p_{V}^{*}$ satisfies homogeneous Neumann boundary conditions on $\Gamma_{N}$ and

$$
p_{V_{D}}^{*}=\lambda^{2} \frac{\partial p_{\rho}^{*}}{\partial \nu} \quad \text { in } H^{1 / 2}\left(\Gamma_{D}\right)^{\prime}
$$

so that the proposition is proved.

From (A8) we infer the variational inequality [10]

$$
\nabla_{u} \mathcal{L}\left(x^{*}, p^{*}\right)\left(u-u^{*}\right) \geq 0 \text { for all } u \in U_{\mathrm{ad}},
$$

which gives

$$
\left\langle D_{\beta} u^{*}+\int_{W} \rho^{*} p_{\rho}^{*} \nabla_{u} B\left(\cdot ; u^{*}\right)^{T} \mathrm{~d} \mathbf{x}, u-u^{*}\right\rangle_{\mathbb{R}^{\ell}} \geq 0 \quad \text { for all } u \in U_{\mathrm{ad}},
$$

where $D_{\beta}=\operatorname{diag}\left(\beta_{1}, \ldots, \beta_{\ell}\right) \in \mathbb{R}^{\ell \times \ell}$ is a diagonal matrix. In particular, if $u^{*}$ is an interior point of $U_{\text {ad }}$ we will obtain the implicit formula

$$
u_{i}^{*}=-\frac{1}{\beta_{i}} \int_{W} \rho^{*} p_{\rho}^{*} \frac{\partial B}{\partial u_{i}}\left(\cdot ; u^{*}\right) \mathrm{d} \mathbf{x} \quad \text { for } i=1, \ldots, \ell
$$

for the components of the optimal control $u^{*}$.

\section{Appendix A.}

A.1. Proof of Theorem 2.4. The proof is divided into several steps.

Step 1. Given $M \in \mathbb{R}^{+}$we introduce the convex set

$$
\mathrm{C}(M)=\left\{F \in L^{2}(W) \mid\|F\|_{L^{\infty}(W)} \leq M\right\},
$$

and we define a mapping $T_{M}: \mathrm{C}(M) \rightarrow H^{1}(W)$ in three steps.

Step 1.1. Given $F \in \mathrm{C}(M)$ we denote by $\rho(F)$ the unique minimizer of

$$
\rho \mapsto E(F)(\rho)=E_{q u}(\rho)+E_{e l}(\rho)+E_{q u p o t}(\rho)-\int_{W} \rho^{2} F \mathrm{~d} \mathbf{x}
$$

in $\rho_{D}+H_{\circ}$. Following [3, Theorem 2.2] we have $\rho(F) \in L^{\infty}(W)$ with $\inf \rho(F)>0$ and the mapping $\rho(\cdot)$ is $L^{2}(W) \rightarrow H^{1}(W)$-continuous.

Step 1.2. Given $\rho \in L^{\infty}(W)$ with $\inf \rho>0$ it is readily seen that

$$
-\nabla \cdot(\rho \nabla F)=0 \text { a.e. in } W, \quad F-F_{D} \in H_{\circ}
$$

has a unique $H^{1}(W)$-weak solution $F_{\circ}(\rho)$ - i.e., there is exactly one $F_{\circ}(\rho)=F \in$ $F_{D}+H_{\circ}$ such that $(2.5 \mathrm{a})$ holds for all $\varphi \in H_{\circ}$. Furthermore, due to $0<\rho<+\infty$ weak minimum/maximum principles allow to deduce inf $F_{D} \leq F_{\circ}(\rho) \leq \sup F_{D}$.

Step 1.3. We set $T_{M}(F)=F_{\circ}(\rho(F))$. Obviously, if $M>\left\|F_{D}\right\|_{L^{\infty}(W)}$, then $T_{M}$ will map $\mathrm{C}(M)$ into $\mathrm{C}(M)$. Furthermore, if $(F, \rho, V)$ is a $H^{1}$ solution of (2.3) with $\operatorname{essinf}_{\mathbf{x} \in D} \rho(\mathbf{x})>0$, then there will be an $M>0-$ e.g. any $M$ with $M>\|F\|_{L^{\infty}(W)}-$ such that $F \in \mathrm{C}(M)$ and $F$ is a fixed point of $T_{M}$.

Step 2. Now we derive an a priori estimate for $V_{\circ}\left(\rho(F)^{2}-C\right)$, where here and in the remaining part of Step 2 we keep $F \in L^{\infty}(W)$ fixed. We recall: $\rho(F)$ is the minimizer of $E(F)$ in $\rho_{D}+H_{\circ}$. Since $\rho_{D} \in \rho_{D}+H_{\circ}$ we have

$$
E(F)(\rho(F)) \leq E(F)\left(\rho_{D}\right)
$$


From $\rho_{D} \in H_{b}$ we conclude that $E(F)\left(\rho_{D}\right)<+\infty$. Furthermore, for all $\rho \in \rho_{D}+H_{\circ}$, we have due to

$$
\forall t, \kappa \in\left[0,+\infty\left[: \quad t \cdot(\log t-1-\kappa) \geq-e^{\kappa},\right.\right.
$$

$$
\begin{aligned}
& E(F)(\rho) \\
= & \varepsilon^{2} \int_{W}|\nabla \rho|^{2} \mathrm{~d} \mathbf{x}+\int_{W} \rho^{2}\left(\log \left(\rho^{2}\right)-1+V^{*}+B-F\right) \mathrm{d} \mathbf{x}+\frac{1}{2} \int_{W}\left|\nabla V_{\circ}\left(\rho^{2}-C\right)\right|^{2} \mathrm{~d} \mathbf{x} \\
\geq & \varepsilon^{2} \int_{W}|\nabla \rho|^{2} \mathrm{~d} \mathbf{x}+\int_{W} \rho^{2}\left(\log \left(\rho^{2}\right)-1-\left\|V^{*}\right\|_{L^{\infty}(W)}-\|B\|_{L^{\infty}(W)}-\|F\|_{L^{\infty}(W)}\right) \mathrm{d} \mathbf{x} \\
\geq & \varepsilon^{2} \int_{W}|\nabla \rho|^{2} \mathrm{~d} \mathbf{x}-\operatorname{meas}(W) \exp \left(\left\|V^{*}\right\|_{L^{\infty}(W)}+\|B\|_{L^{\infty}(W)}+\|F\|_{L^{\infty}(W)}\right),
\end{aligned}
$$

where meas $(W)$ is the Lebesgue measure of $W$. Utilizing

$$
\|\varphi\|_{L^{\infty}(W)} \leq\|\varphi\|_{H \cap L^{\infty}(W)} \text { for all } \varphi \in H^{1} \cap L^{\infty}(W)
$$

and $\left\|V_{*}\right\|_{L^{\infty}(W)} \leq\left\|V_{D}\right\|_{L^{\infty}(W)} \leq\left\|V_{D}\right\|_{b}$ we obtain

$$
\begin{aligned}
& E(F)(\rho) \\
\geq & \varepsilon^{2} \int_{W}|\nabla \rho|^{2} \mathrm{~d} \mathbf{x}-\operatorname{meas}(W) \exp \left(\left\|V_{D}\right\|_{b}+\|B\|_{L^{\infty}(W)}+\|F\|_{L^{\infty}(W)}\right) \\
= & \varepsilon^{2} \int_{W}|\nabla \rho|^{2} \mathrm{~d} \mathbf{x}-c_{1} e^{\|B\|_{L^{\infty}(W)}+\|F\|_{L^{\infty}(W)},}
\end{aligned}
$$

where the constant $c_{1}>0$ depends on meas $(W),\left\|V_{D}\right\|_{H_{b}}$, such that via (A.1),

$$
\int_{W}|\nabla \rho(F)|^{2} \mathrm{~d} \mathbf{x} \leq \frac{1}{\varepsilon^{2}}\left(E(F)\left(\rho_{D}\right)+c_{1} e^{\|B\|_{L^{\infty}(W)}+\|F\|_{L}(W)}\right) .
$$

In order to obtain an upper estimate for $E(F)\left(\rho_{D}\right)$ we calculate via Remark 2.3-e) and via

$$
\forall t \in\left[0,+\infty\left[: \quad \log t \leq 1+t, \quad t \cdot(\log t-1) \leq t^{2},\right.\right.
$$

$$
\begin{aligned}
& E(F)\left(\rho_{D}\right) \\
= & \varepsilon^{2} \int_{W}\left|\nabla \rho_{D}\right|^{2} \mathrm{~d} \mathbf{x}+\int_{W} \rho_{D}^{2}\left(\log \left(\rho_{D}^{2}\right)-1+V^{*}+B-F\right) \mathrm{d} \mathbf{x}+\frac{1}{2} \int_{W}\left|\nabla V_{\circ}\left(\rho_{D}^{2}-C\right)\right|^{2} \mathrm{~d} \mathbf{x} \\
\leq & \varepsilon^{2}\left\|\rho_{D}\right\|_{H^{1}(W)}^{2}+\int_{W}\left\|\rho_{D}\right\|_{L^{\infty}(W)}^{4} \mathrm{~d} \mathbf{x} \\
& +\int_{W}\left\|\rho_{D}\right\|_{L^{\infty}(W)}^{2}\left(\left\|V^{*}\right\|_{L^{\infty}(W)}+\|B\|_{L^{\infty}(W)}+\|F\|_{L^{\infty}(W)}\right) \mathrm{d} \mathbf{x} \\
& +\frac{C_{P} \operatorname{meas}(W)}{2 \lambda^{4}}\left(\left\|\rho_{D}\right\|_{L^{\infty}(W)}^{2}+\|C\|_{L^{\infty}(W)}\right)^{2} \\
\leq & \varepsilon^{2}\left\|\rho_{D}\right\|_{b}^{2}+\int_{W}\left\|\rho_{D}\right\|_{b}^{4} \mathrm{~d} \mathbf{x} \\
& +\int_{W}\left\|\rho_{D}\right\|_{b}^{2}\left(\left\|V_{D}\right\|_{b}+\|B\|_{L^{\infty}(W)}+\|F\|_{b}\right) \mathrm{d} \mathbf{x} \\
& +\frac{C_{P} \operatorname{meas}(W)}{2 \lambda^{4}}\left(\left\|\rho_{D}\right\|_{b}^{2}+\|C\|_{L^{\infty}(W)}\right)^{2},
\end{aligned}
$$


i.e., there is a constant $c_{2}>0$ depending on meas $(W), \varepsilon, \lambda, C_{P},\left\|\rho_{D}\right\|_{b},\left\|V_{D}\right\|_{b}$, $\|C\|_{L^{\infty}(W)}$ with

$$
\begin{aligned}
E(F)\left(\rho_{D}\right) & \leq c_{2}\left(1+\|B\|_{L^{\infty}(W)}+\|F\|_{L^{\infty}(W)}\right) \\
& \leq c_{2} e^{\|B\|_{L^{\infty}(W)}+\|F\|_{L^{\infty}(W)}}
\end{aligned}
$$

With the aid of Poincaré's inequality we deduce from (A.2), (A.3),

$$
\begin{aligned}
& \|\rho(F)\|_{H^{1}(W)} \leq\left\|\rho(F)-\rho_{D}\right\|_{H^{1}(W)}+\left\|\rho_{D}\right\|_{H^{1}(W)} \\
& \leq \sqrt{1+C_{P}} \sqrt{\int_{W}\left|\nabla\left(\rho(F)-\rho_{D}\right)\right|^{2} \mathrm{~d} \mathbf{x}}+\left\|\rho_{D}\right\|_{b} \\
& \leq \sqrt{1+C_{P}} \sqrt{\int_{W}|\nabla \rho(F)|^{2} \mathrm{~d} \mathbf{x}+\int_{W}\left|\nabla \rho_{D}\right|^{2} \mathrm{~d} \mathbf{x}}+\left\|\rho_{D}\right\|_{b} \\
& \leq \sqrt{1+C_{P}}\left(\sqrt{\int_{W}|\nabla \rho(F)|^{2} \mathrm{~d} \mathbf{x}}+\sqrt{\int_{W}\left|\nabla \rho_{D}\right|^{2} \mathrm{~d} \mathbf{x}}\right)+\left\|\rho_{D}\right\|_{b} \\
& \leq \sqrt{1+C_{P}} \sqrt{\int_{W}|\nabla \rho(F)|^{2} \mathrm{~d} \mathbf{x}}+\left(1+\sqrt{1+C_{P}}\right)\left\|\rho_{D}\right\|_{b}
\end{aligned}
$$

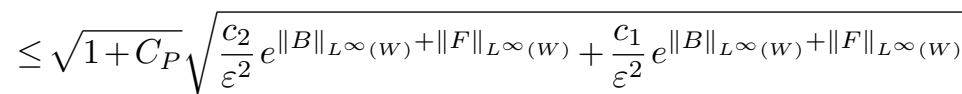

$$
\begin{aligned}
& +\left(1+\sqrt{1+C_{P}}\right)\left\|\rho_{D}\right\|_{b},
\end{aligned}
$$

i.e., there is a constant $c_{3}>0$ depending on meas $(W), \varepsilon, \lambda, C_{P},\left\|\rho_{D}\right\|_{b},\left\|V_{D}\right\|_{b}$, $\|C\|_{L^{\infty}(W)}$ such that

$$
\|\rho(F)\|_{H^{1}(W)} \leq c_{3} e^{\|B\|_{L^{\infty}(W)}+\|F\|_{L \infty}(W)} .
$$

Following [1] there is a constant $c_{4}>0$, just depending on $W$ such that due to $d=1,2$ or $d=3$,

$$
\|\rho(F)\|_{L^{6}(W)} \leq c_{4}\|\rho(F)\|_{H^{1}(W)},
$$

and due to [5] there is a constant $c_{5}>0$, just depending on $W$ such that

$$
\left\|V_{\circ}\left(\rho(F)^{2}-C\right)\right\|_{L^{\infty}(W)} \leq \frac{c_{5}}{\lambda^{2}}\left\|\rho(F)^{2}-C\right\|_{L^{3}(W)} .
$$

We deduce from (A.5) and (A.4),

$$
\begin{aligned}
\left\|V_{\circ}\left(\rho(F)^{2}-C\right)\right\|_{L^{\infty}(W)} & \leq \frac{c_{5}}{\lambda^{2}}\left(\|\rho\|_{L^{6}(W)}^{2}+\operatorname{meas}(W)^{1 / 3}\|C\|_{L^{\infty}(W)}\right) \\
& \leq \frac{c_{5}}{\lambda^{2}}\left(c_{4}\|\rho(F)\|_{H^{1}(W)}^{2}+\operatorname{meas}(W)^{1 / 3}\|C\|_{L^{\infty}(W)}\right) \\
& \leq \frac{c_{5}}{\lambda^{2}}\left(c_{4}^{2} c_{3}^{2} e^{\left.2\|B\|_{L^{\infty}(W)}+2\|F\|_{L^{\infty}(W)}+\operatorname{meas}(W)^{1 / 3}\|C\|_{L^{\infty}(W)}\right),}\right.
\end{aligned}
$$

i.e., there exists a constant $c_{6} \in \mathbb{R}^{+}$depending on $W, \varepsilon, \lambda, C_{P},\left\|\rho_{D}\right\|_{b},\left\|V_{D}\right\|_{b}$, $\|C\|_{L^{\infty}(W)}$ such that

$$
\left\|V_{\circ}\left(\rho(F)^{2}-C\right)\right\|_{L^{\infty}(W)} \leq c_{6} e^{2\|B\|_{L^{\infty}(W)}+2\|F\|_{L^{\infty}(W)} .}
$$


Step 3. Now we derive an a priori estimate on $\|\rho(F)\|_{L^{\infty}(W)}$, where throughout Step 3 we assume that $F \in L^{\infty}(W)$ is fixed. Due to Theorem 2.2 in [3] the minimizer $\rho=\rho(F)$ satisfies $(2.5 \mathrm{~b})$ for all $\varphi \in H_{\circ}$. A standard monotonicity argument yields the estimates

$$
\log \left((\sup \rho(F))^{2}\right)-\|V\|_{L^{\infty}(W)}-\|B\|_{L^{\infty}(W)}-\|F\|_{L^{\infty}(W)} \leq 0
$$

and

$$
\log \left((\inf \rho(F))^{2}\right)+\|V\|_{L^{\infty}(W)}+\|B\|_{L^{\infty}(W)}+\|F\|_{L^{\infty}(W)} \geq 0,
$$

such that via $V=V_{\circ}\left(\rho(F)^{2}-C\right)+V^{*}$ and via (A.6),

$$
\begin{aligned}
\log \left((\sup \rho(F))^{2}\right) \leq & \left\|V^{*}\right\|_{L^{\infty}(W)}+c_{6} e^{2\|B\|_{L^{\infty}(W)}+2\|F\|_{L^{\infty}(W)}}+\|B\|_{L^{\infty}(W)} \\
& +\|F\|_{L^{\infty}(W)}, \\
\log \left((\inf \rho(F))^{2}\right) \geq & -\left\|V^{*}\right\|_{L^{\infty}(W)}-c_{6} e^{2\|B\|_{L^{\infty}(W)}+2\|F\|_{L^{\infty}(W)}-\|B\|_{L^{\infty}(W)}} \\
& -\|F\|_{L^{\infty}(W)},
\end{aligned}
$$

i.e., there is a constant $c_{7}>0$ depending on $W, \varepsilon, \lambda, C_{P},\left\|\rho_{D}\right\|_{b},\left\|V_{D}\right\|_{b},\|C\|_{L^{\infty}(W)}$ such that

$$
\exp \left(-c_{7} e^{2\|B\|_{L^{\infty}(W)}+2\|F\|_{L^{\infty}(W)}}\right) \leq \rho(F) \leq \exp \left(c_{7} e^{2\|B\|_{L^{\infty}(W)}+2\|F\|_{L^{\infty}(W)}}\right) .
$$

Step 4. We follow the lines of the proofs of Theorems 2.1, 2.2 and Lemma 2.3 in [3] to deduce that $T_{M}: \mathrm{C}(M) \rightarrow H^{1}(W)$ is $L^{2}(W) \rightarrow L^{2}(W)$-continuous and $T_{M}$ maps the convex set $\mathrm{C}(M)$ - which is closed in $L^{2}(W)$ - into $H^{1}(W)$ which is precompact in $L^{2}(W)$. Furthermore, if $M>\|F\|_{L^{\infty}(W)}$, then - due to the lower estimate on $\rho(F)$ according to (A.7), see Step $1.3-T_{M}$ maps $\mathrm{C}(M)$ into $\mathrm{C}(M)$. By Schauder's Fixed Point Theorem, $T_{M}$ has in this case a fixed point. Certainly, any fixed point of $T_{M}$ is a $H^{1}$ solution of (2.3). Thus, (2.3) has at least one $H^{1}$ solution.

Step 5. Now let $(F, \rho, V)$ be an arbitrary $H^{1}$ solution of $(2.3)$. Then there is $M \in \mathbb{R}^{+}$ with $F \in T_{M}$ and we have $\rho=\rho(F)$. By (A.7),

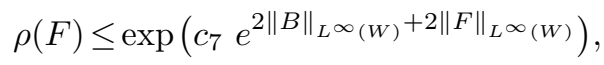

by (A.4),

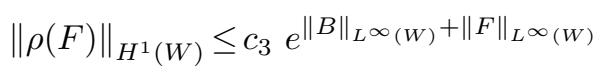

by (A.6),

$$
\begin{aligned}
\|V\|_{L^{\infty}(W)} & =\left\|V_{\circ}\left(\rho(F)^{2}-C\right)+V^{*}\right\|_{L^{\infty}(W)} \\
& \leq c_{6} e^{\|B\|_{L^{\infty}(W)}+\|F\|_{L^{\infty}(W)}}+\left\|V_{D}\right\|_{L^{\infty}(W)},
\end{aligned}
$$

and by easy manipulations of Poisson's equation there is $c_{8}>0$ depending on $W, \lambda$, $C_{P},\|C\|_{L^{\infty}(W)}$ such that

$$
\left\|V_{\circ}\left(\rho(F)^{2}-C\right)\right\|_{H^{1}(W)} \leq c_{8}\left(1+\|\rho(F)\|_{L^{\infty}(W)}^{2}\right),
$$

and due to

$$
\|F\|_{L^{\infty}(W)} \leq\left\|F_{D}\right\|_{L^{\infty}(W)} \leq\left\|F_{D}\right\|_{b}
$$


and due to a standard argument we deduce

$$
\sqrt{\int_{W}\left|\nabla\left(F-F_{D}\right)\right|^{2} \mathrm{~d} \mathbf{x}} \leq \frac{\operatorname{esssup} \rho(F)}{\operatorname{essinf} \rho(F)} \sqrt{\int_{W}\left|\nabla F_{D}\right|^{2} \mathrm{~d} \mathbf{x}}
$$

Consequence: there is a constant $c \in \mathbb{R}^{+}$depending on $W, \varepsilon, \lambda, C_{P},\left\|\rho_{D}\right\|_{b},\left\|V_{D}\right\|_{b}$, $\|C\|_{L^{\infty}(W)},\left\|F_{D}\right\|_{b}$ such that

$$
\|F\|_{b}+\|\rho\|_{b}+\|V\|_{b} \leq c e^{2 e^{\|B\|_{L} \infty(W)}}
$$

which gives (2.6).

A.2. Proof of Lemma 3.2. We start by computing formally the first and second directional derivative for $I$ at $(\bar{F}, \bar{\rho}) \in H_{b} \times H_{b}$ in directions $(F, \rho),(\tilde{F}, \tilde{\rho}) \in H_{b} \times$ $H_{b}$ and find

$$
\nabla I(\bar{F}, \bar{\rho})(F, \rho)=-\frac{q}{L_{1}} \int_{W}\left(2 \bar{\rho} \rho \nabla \bar{F}+\bar{\rho}^{2} \nabla F\right) \cdot \vec{e}_{1} \mathrm{~d} \mathbf{x}
$$

and

$$
\nabla^{2} I(\bar{F}, \bar{\rho})((F, \rho),(\tilde{F}, \tilde{\rho}))=-\frac{2 q}{L_{1}} \int_{W}(\tilde{\rho} \rho \nabla \bar{F}+\bar{\rho} \rho \nabla \tilde{F}+\bar{\rho} \tilde{\rho} \nabla F) \cdot \vec{e}_{1} \mathrm{~d} \mathbf{x}
$$

To prove that both directional derivatives are Fréchet-derivatives we estimate

$$
\begin{aligned}
& |I(\bar{F}+F, \bar{\rho}+\rho)-I(\bar{F}, \bar{\rho})-\nabla I(\bar{F}, \bar{\rho})(F, \rho)| \\
\leq & \frac{q}{L_{1}} \int_{W}\left|\left(\rho^{2} \nabla \bar{F}+\left(2 \bar{\rho} \rho+\rho^{2}\right) \nabla F\right) \cdot \vec{e}_{1}\right| \mathrm{d} \mathbf{x} \\
\leq & c_{1}\left(\|\rho\|_{L^{4}(W)}^{2}\|\nabla \bar{F}\|_{L^{2}(W)}+\|\rho\|_{L^{4}(W)}\left(1+2\|\bar{\rho}\|_{L^{4}(W)}\|\rho\|_{L^{4}(W)}\right)\|\nabla F\|_{L^{2}(W)}\right),
\end{aligned}
$$

where we have introduced the positive constant $c_{1}=q\left\|\vec{e}_{1}\right\|_{L^{2}(W)} / L_{1}$. Utilizing Young's inequality and the continuous embedding of $L^{\infty}(W)$ in $L^{4}(W)$ there exists a constant $c_{2}>0$ depending on $c_{1},\|\nabla \bar{F}\|_{L^{2}(W)}$, and $\|\bar{\rho}\|_{L^{4}(W)}$ such that

$$
|I(\bar{F}+F, \bar{\rho}+\rho)-I(\bar{F}, \bar{\rho})-\nabla I(\bar{F}, \bar{\rho})(F, \rho)| \leq c_{2}\left(\|\rho\|_{H_{b}}^{2}+\|\rho\|_{H_{b}}^{4}+\|F\|_{H_{b}}^{2}\right) .
$$

Consequently,

$$
\lim _{\|(F, \rho)\|_{H_{b} \times H_{b}} \rightarrow 0} \frac{|I(\bar{F}+F, \bar{\rho}+\rho)-I(\bar{F}, \bar{\rho})-\nabla I(\bar{F}, \bar{\rho})(F, \rho)|}{\|(F, \rho)\|_{H_{b} \times H_{b}}}=0 .
$$

Thus, the first directional derivative of $I$ given by (A.8) is its first Fréchet-derivative. Now we turn to the second derivative. Analogous to the first derivative we estimate

$$
\begin{aligned}
& \left|\nabla I(\bar{F}+\tilde{F}, \bar{\rho}+\tilde{\rho})(F, \rho)-\nabla I(\bar{F}, \bar{\rho})(F, \rho)-\nabla^{2} I(\bar{F}, \bar{\rho})((F, \rho),(\tilde{F}, \tilde{\rho}))\right| \\
\leq & \frac{q}{L_{1}} \int_{W}\left|\left(2 \rho \tilde{\rho} \nabla \tilde{F}+(\tilde{\rho})^{2} \nabla F\right) \cdot \vec{e}_{1}\right| \mathrm{d} \mathbf{x} \\
\leq & \frac{q\left\|\vec{e}_{1}\right\|_{L^{2}(W)}}{L_{1}}\left(2\|\rho\|_{L^{4}(W)}\|\tilde{\rho}\|_{L^{4}(W)}\|\nabla \tilde{F}\|_{L^{2}(W)}+\|\tilde{\rho}\|_{L^{4}(W)}^{2}\|\nabla F\|_{L^{2}(W)}\right) \\
\leq & \frac{q\left\|\vec{e}_{1}\right\|_{L^{2}(W)}}{L_{1}}\left(\|\rho\|_{L^{4}(W)}^{2}\|\tilde{\rho}\|_{L^{4}(W)}^{2}+\|\nabla \tilde{F}\|_{L^{2}(W)}^{2}+\|\tilde{\rho}\|_{L^{4}(W)}^{2}\|\nabla F\|_{L^{2}(W)}\right),
\end{aligned}
$$


where we have used Young's inequality. Since $H_{b}$ is continuously (even compactly) embedded in $L^{4}(W)$, there exists a constant $c_{3}>0$ depending on $q,\left\|\vec{e}_{1}\right\|_{L^{2}(W)}, L_{1}$, $\|\rho\|_{L^{4}(W)}$, and $\|\nabla F\|_{L^{2}(W)}$ such that

$$
\begin{aligned}
& \left|\nabla I(\bar{F}+\tilde{F}, \bar{\rho}+\tilde{\rho})(F, \rho)-\nabla I(\bar{F}, \bar{\rho})(F, \rho)-\nabla^{2} I(\bar{F}, \bar{\rho})((F, \rho),(\tilde{F}, \tilde{\rho}))\right| \\
\leq & c_{3}\|(\tilde{F}, \tilde{\rho})\|_{H_{b} \times H_{b}}^{2} .
\end{aligned}
$$

Hence

$$
\lim _{\|(\tilde{F}, \tilde{\rho})\|_{H_{b} \times H_{b} \rightarrow 0}} \frac{\left\|\nabla I(\bar{F}+\tilde{F}, \bar{\rho}+\tilde{\rho})-\nabla I(\bar{F}, \bar{\rho})-\nabla^{2} I(\bar{F}, \bar{\rho})(\tilde{F}, \tilde{\rho})\right\|_{H_{b}^{\prime}}}{\|(\tilde{F}, \tilde{\rho})\|_{H_{b} \times H_{b}}}=0 .
$$

We conclude that the second directional derivative of $I$ is the second Fréchetderivative.

To prove continuity of the mapping $(\bar{F}, \bar{\rho}) \mapsto \nabla^{2} I(\bar{F}, \bar{\rho})$ in the operator norm let us consider $\left(\bar{F}_{1}, \bar{\rho}_{1}\right),\left(\bar{F}_{2}, \bar{\rho}_{2}\right) \in H_{b} \times H_{b}$. Due to linearity and by similar arguments as used before we obtain for all $(F, \rho),(\tilde{F}, \tilde{\rho}) \in H_{b} \times H_{b}$ the estimate

$$
\begin{aligned}
& \left|\nabla^{2} I\left(\bar{F}_{1}, \bar{\rho}_{1}\right)((F, \rho),(\tilde{F}, \tilde{\rho}))-\nabla^{2} I\left(\bar{F}_{2}, \bar{\rho}_{2}\right)((F, \rho),(\tilde{F}, \tilde{\rho}))\right| \\
\leq & \frac{q}{L_{1}}\left\|\nabla \bar{F}_{1}-\nabla \bar{F}_{2}\right\|_{L^{2}(W)}\|\rho\|_{L^{4}(W)}\|\tilde{\rho}\|_{L^{4}(W)} \\
& +\frac{q}{L_{1}}\left\|\bar{\rho}_{1}-\bar{\rho}_{2}\right\|_{L^{4}(W)}\left(\|\rho\|_{L^{4}(W)}\|\nabla \tilde{F}\|_{L^{2}(W)}+\|\tilde{\rho}\|_{L^{4}(W)}\|\nabla F\|_{L^{2}(W)}\right) .
\end{aligned}
$$

We deduce via $\bar{\rho}_{1}, \bar{\rho}_{2} \in L^{\infty}(W)$ that there is a constant $c_{4}>0$ only depending on $W$ such that

$$
\begin{aligned}
& \sup \left\{\left|\nabla^{2} I\left(\bar{F}_{1}, \bar{\rho}_{1}\right)((F, \rho),(\tilde{F}, \tilde{\rho}))-\nabla^{2} I\left(\bar{F}_{1}, \bar{\rho}_{1}\right)((F, \rho),(\tilde{F}, \tilde{\rho}))\right|:\right. \\
& \left.(F, \rho),(\tilde{F}, \tilde{\rho}) \in H_{b} \times H_{b} \text { with }\|(F, \rho)\|_{H_{b} \times H_{b}},\|(\tilde{F}, \tilde{\rho})\|_{H_{b} \times H_{b}} \leq 1\right\} \\
\leq & c_{4}\left(\left\|\nabla \bar{F}_{1}-\nabla \bar{F}_{2}\right\|_{L^{2}(W)}+\left\|\bar{\rho}_{1}-\bar{\rho}_{2}\right\|_{L^{\infty}(W)}\right) \leq c_{4}\left\|\left(\bar{F}_{1}, \bar{\rho}_{1}\right)-\left(\bar{F}_{2}, \bar{\rho}_{2}\right)\right\|_{H_{b} \times H_{b}} .
\end{aligned}
$$

Hence, $\nabla^{2} I$ is a (Lipschitz)-continuous function and the claim is proved.

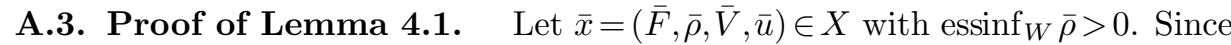
$e^{4}, e^{5}, e^{6}$ are linear and bounded on $X$, these operators are Fréchet-differentiable. In fact, for directions $x=(F, \rho, V, u) \in X$ with $\operatorname{essinf}_{W}(\bar{\rho}+\rho)>0$ we find

$$
\nabla e^{4}(\bar{x}) x=\tau_{D}(F), \quad \nabla e^{5}(\bar{x}) x=\tau_{D}(\rho), \quad \nabla e^{6}(\bar{x}) x=\tau_{D}(V) .
$$

Moreover, their second derivatives are zero. Let us turn to the operator $e^{1}$ whose first directional derivative at $\bar{x}$ in direction $x$ is

$$
\left\langle\nabla e^{1}(\bar{x}) x, \varphi\right\rangle_{H_{\circ}^{\prime}, H_{\circ}}=\int_{W}\left(2 \bar{\rho} \rho \nabla \bar{F}+\bar{\rho}^{2} \nabla F\right) \cdot \nabla \varphi \mathrm{d} \mathbf{x} \quad \text { for } \varphi \in H_{\circ} .
$$


Consequently,

$$
\begin{aligned}
& \left\|e^{1}(\bar{x}+x)-e^{1}(\bar{x})-\nabla e^{1}(\bar{x}) x\right\|_{H^{\prime}} \\
= & \sup _{\|\varphi\|_{H_{\circ}}=1}\left|\int_{W}\left(\rho^{2}(\nabla \bar{F}+\nabla F)+2 \bar{\rho} \rho \nabla F\right) \cdot \nabla \varphi \mathrm{d} \mathbf{x}\right| \\
\leq & \sup _{\|\varphi\|_{H_{\circ}}=1}\|\rho\|_{L^{\infty}(W)}^{2}\left(\|\nabla \bar{F}\|_{L^{2}(W)}+\|\nabla F\|_{L^{2}(W)}\right)\|\nabla \varphi\|_{L^{2}(W)} \\
& +2 \sup _{\|\varphi\|_{H_{\circ}}=1}\|\bar{\rho}\|_{L^{\infty}(W)}\|\rho\|_{L^{\infty}(W)}\|\nabla F\|_{L^{2}(W)}\|\nabla \varphi\|_{L^{2}(W)} \\
\leq & \|\rho\|_{H_{b}}^{2}\left(\|\nabla \bar{F}\|_{L^{2}(W)}+\|F\|_{H_{b}}\right)+2\|\bar{\rho}\|_{L^{\infty}(W)}\|\rho\|_{H_{b}}\|F\|_{H_{b}} \\
\leq & c_{1}\left(\|\rho\|_{H_{b}}^{2}\left(1+\|F\|_{H_{b}}\right)+\|\rho\|_{H_{b}}\|F\|_{H_{b}}\right),
\end{aligned}
$$

where the constant $c_{1}>0$ depends on $\|\nabla \bar{F}\|_{L^{2}(W)}$ and $\|\bar{\rho}\|_{L^{\infty}(W)}$. Thus, we have

$$
\lim _{\|x\|_{X} \rightarrow 0} \frac{\left\|e^{1}(\bar{x}+x)-e^{1}(\bar{x})-\nabla e^{1}(\bar{x}) x\right\|_{H^{\prime}}}{\|x\|_{X}}=0 .
$$

The directional derivative of $\nabla e^{1}(\bar{x}) x$ at $\bar{x}$ in direction $\tilde{x}=(\tilde{F}, \tilde{\rho}, \tilde{V}, \tilde{u}) \in X$, is

$$
\left.\left\langle\nabla^{2} e^{1}(\bar{x})(x, \tilde{x}), \varphi\right\rangle_{H_{\circ}^{\prime}, H_{\circ}}=2 \int_{W}(\tilde{\rho} \rho \nabla \bar{F}+\bar{\rho} \rho \nabla \tilde{F}+\bar{\rho} \tilde{\rho} \nabla F)\right) \cdot \nabla \varphi \mathrm{d} \mathbf{x} \quad \text { for } \varphi \in H_{\circ} .
$$

We calculate

$$
\begin{aligned}
& \left\|\nabla e^{1}(\bar{x}+\tilde{x})-\nabla e^{1}(\bar{x})-\nabla^{2} e^{1}(\bar{x})(\tilde{x}, \cdot)\right\|_{L\left(X, H^{\prime}\right)} \\
= & \sup _{\|x\|_{X}=1}\left\|\left(\nabla e^{1}(\bar{x}+\tilde{x})\right) x-\nabla e^{1}(\bar{x}) x-\nabla^{2} e^{1}(\bar{x})(\tilde{x}, x)\right\|_{H^{\prime}} \\
= & \sup _{\|x\|_{X}=1\|\varphi\|_{H_{\circ}}=1} \sup _{1}\left|\left\langle\nabla e^{1}(\bar{x}+\tilde{x}) x-\nabla e^{1}(\bar{x}) x-\nabla^{2} e^{1}(\bar{x})(x, \tilde{x}), \varphi\right\rangle_{H_{\circ}^{\prime}, H_{\circ}}\right| \\
= & \sup _{\|x\|_{X}=1\|\varphi\|_{H_{\circ}}=1} \sup _{\mid}\left|\int_{W} 2\left(\tilde{\rho} \rho \nabla \tilde{F}+\tilde{\rho}^{2} \nabla F\right) \cdot \nabla \varphi \mathrm{d} \mathbf{x}\right| \\
\leq & 2 \sup _{\|x\|_{X}=1}\left(\|\tilde{\rho}\|_{L^{\infty}(W)}\|\rho\|_{L^{\infty}(W)}\|\nabla \tilde{F}\|_{L^{2}\left(W ; \mathbb{R}^{d}\right)}+\|\tilde{\rho}\|_{L^{\infty}(W)}^{2}\|\nabla F\|_{L^{2}\left(W ; \mathbb{R}^{d}\right)}\right) \\
\leq & c_{2}\|\tilde{x}\|_{X}^{2}
\end{aligned}
$$

for a constant $c_{2}>0$, which depends on embedding constants of $L^{\infty}(W), L^{4}(W)$ in $H_{b}$. Here, $L\left(X, H^{\prime}\right)$ denotes the normed linear space of all bounded linear operators from $X$ to $H^{\prime}$ supplied with the common norm. Thus,

$$
\lim _{\|\tilde{x}\|_{X} \rightarrow 0} \frac{\left\|\nabla e^{1}(\bar{x}+\tilde{x})-\nabla e^{1}(\bar{x})-\nabla^{2} e^{1}(\bar{x})(\tilde{x}, \cdot)\right\|_{L\left(X, H^{\prime}\right)}}{\|\tilde{x}\|_{X}}=0 .
$$

Since the mapping $\bar{x} \mapsto \nabla^{2} e^{1}(\bar{x})$ is linear and bounded, we conclude that $\nabla^{2} e^{1}$ is Lipschitz-continuous.

We proceed by investigating the operator $e^{2}$. The first directional derivative has the 
form

$$
\begin{aligned}
\left\langle\nabla e^{2}(\bar{x}) x, \varphi\right\rangle_{H_{\circ}^{\prime}, H_{\circ}}= & \int_{W} \rho\left(\bar{V}+B(\cdot ; \bar{u})+\log \left((\bar{\rho})^{2}\right)-\bar{F}\right) \varphi \mathrm{d} \mathbf{x} \\
& +\int_{W} \varepsilon \nabla \rho \cdot \nabla \varphi+\left(\bar{\rho}\left(V+\nabla_{u} B(\cdot ; \bar{u}) u-F\right)+2 \rho\right) \varphi \mathrm{d} \mathbf{x}
\end{aligned}
$$

for $\varphi \in H_{\circ}$. We derive

$$
\begin{aligned}
& \left\|e^{2}(\bar{x}+x)-e^{2}(\bar{x})-\nabla e^{2}(\bar{x}) x\right\|_{H^{\prime}} \\
& \leq \sup _{\|\varphi\|_{H_{0}}=1} \int_{W}[(\rho V+\rho(B(\cdot ; \bar{u}+u)-B(\cdot ; \bar{u}))+2 \rho(\log (\bar{\rho}+\rho)-\log (\bar{\rho}))) \varphi \\
& +\left(-\rho F+\bar{\rho}\left(B(\cdot ; \bar{u}+u)-B(\cdot ; \bar{u})-\nabla_{u} B(\cdot ; \bar{u}) u\right) \varphi\right. \\
& \left.+2 \bar{\rho}\left(\log (\bar{\rho}+\rho)-\log (\bar{\rho})-\frac{\rho}{\bar{\rho}}\right) \varphi\right] \mathrm{d} \mathbf{x} \\
& \leq\|\rho\|_{L^{\infty}(W)}\left(\|V\|_{L^{2}(W)}+\|B(\cdot ; \bar{u}+u)-B(\cdot ; \bar{u})\|_{L^{2}(W)}\right) \\
& +\|\rho\|_{L^{\infty}(W)}\left(2\|\log (\bar{\rho}+\rho)-\log (\bar{\rho})\|_{L^{2}(W)}+\|F\|_{L^{\infty}(W)}\right) \\
& +\|\bar{\rho}\|_{L^{\infty}(W)}\left\|B(\cdot ; \bar{u}+u)-B(\cdot ; \bar{u})-\nabla_{u} B(\cdot ; \bar{u}) u\right\|_{L^{2}(W)} \\
& +2\|\bar{\rho}\|_{L^{\infty}(W)}\left\|\log (\bar{\rho}+\rho)-\log (\bar{\rho})-\frac{\rho}{\bar{\rho}}\right\|_{L^{2}(W)} .
\end{aligned}
$$

By (A7) the mapping $B$ is twice continuously differentiable with respect to the control variable. Thus, there exist constants $c_{2}, c_{3}>0$ depending on $\bar{u}$ such that

$$
\begin{array}{r}
\|B(\cdot ; \bar{u}+u)-B(\cdot ; \bar{u})\|_{L^{2}(W)} \leq c_{2}\|u\|_{\mathbb{R}^{\ell}} \\
\left\|B(\cdot ; \bar{u}+u)-B(\cdot ; \bar{u})-\nabla_{u} B(\cdot ; \bar{u}) u\right\|_{L^{2}(W)} \leq c_{3}\|u\|_{\mathbb{R}^{\ell}}^{2} .
\end{array}
$$

Since $\bar{\rho}, \rho \in H_{b}$ with $\operatorname{essinf}_{W} \bar{\rho}>0$ and $\operatorname{essinf}_{W}(\bar{\rho}+\rho)>0$ there exist constant $c_{3}, c_{4}>0$ depending on $\bar{\rho}$ such that

$$
\begin{aligned}
\|\log (\bar{\rho}+\rho)-\log (\bar{\rho})\|_{L^{2}(W)} & \leq c_{4}\|\rho\|_{H_{b}}, \\
\left\|\log (\bar{\rho}+\rho)-\log (\bar{\rho})-\frac{\rho}{\bar{\rho}}\right\|_{L^{2}(W)} & \leq c_{5}\|\rho\|_{H_{b}}^{2} .
\end{aligned}
$$

From (A.10) and $\|\varphi\|_{L^{\infty}(W)} \leq\|\varphi\|_{H_{b}}$ for all $\varphi \in H_{b}$ it follows that

$$
\begin{aligned}
\left\|e^{2}(\bar{x}+x)-e^{2}(\bar{x})-\nabla e^{2}(\bar{x}) x\right\|_{H^{\prime}} \leq & c_{6}\|\rho\|_{H_{b}}\left(\|V\|_{H_{b}}+\|u\|_{\mathbb{R}^{\ell}}+\|\rho\|_{H_{b}}+\|F\|_{H_{b}}\right) \\
& +c_{6}\|\bar{\rho}\|_{H_{b}}\left(\|u\|_{\mathbb{R}^{\ell}}^{2}+\|\bar{\rho}\|_{H_{b}}\|\rho\|_{H_{b}}^{2}\right),
\end{aligned}
$$

where $c_{6}=\max \left\{1, c_{2}, c_{3} 2 c_{4}, 2 c_{5}\right\}>0$. Using Young's inequality there is a constant $c_{7}>0$ depending on $c_{6}$ and $\bar{\rho}$ satisfying

$$
\left\|e^{2}(\bar{x}+x)-e^{1}(\bar{x})-\nabla e^{2}(\bar{x}) x\right\|_{H^{\prime}} \leq c_{7}\|x\|_{X}^{2}
$$

that implies

$$
\lim _{\|x\|_{X} \rightarrow 0} \frac{\left\|e^{2}(\bar{x}+x)-e^{2}(\bar{x})-\nabla e^{2}(\bar{x}) x\right\|_{H^{\prime}}}{\|x\|_{X}}=0 .
$$


Hence, the the first directional derivative of $e^{2}$ is already the Fréchet-derivative. Next we turn to the second directional derivative of $e^{2}$ at $\bar{x}$. In direction $x=(F, \rho, V, u), \tilde{x}=$ $(\tilde{F}, \tilde{\rho}, \tilde{V}, \tilde{u}) \in X$ with $\operatorname{essinf}_{W}(\bar{\rho}+\tilde{\rho})>0$, we find

$$
\begin{aligned}
\left\langle\nabla^{2} e^{2}(\bar{x})(\tilde{x}, x), \varphi\right\rangle_{H_{\circ}^{\prime}, H_{\circ}}= & \int_{W}\left(\rho\left(\tilde{V}+\nabla_{u} B(\cdot ; \bar{u}) \tilde{u}+\frac{2 \tilde{\rho}}{\bar{\rho}}-\tilde{F}\right)+\tilde{\rho} V\right) \varphi \mathrm{d} \mathbf{x} \\
& +\int_{W}\left(\tilde{\rho}\left(\nabla_{u} B(\cdot ; \bar{u}) u-F+\tilde{u}^{T} \nabla_{u u} B(\cdot ; \bar{u}) u \bar{\rho}\right)\right) \varphi \mathrm{d} \mathbf{x}
\end{aligned}
$$

for $\varphi \in H_{\circ}$. Then, we obtain

$$
\begin{aligned}
& \left\|\nabla e^{2}(x+\tilde{x})-\nabla e^{2}(\bar{x})-\nabla^{2} e^{2}(\bar{x})(\tilde{x}, \cdot)\right\|_{L\left(X, H^{\prime}\right)} \\
& =\sup _{\|x\|_{X}=1\|\varphi\|_{H_{\circ}}=1} \sup _{W} \rho\left(B(\cdot ; \bar{u}+\tilde{u})-B(\cdot ; \bar{u})-\nabla_{u} B(\cdot ; \bar{u}) \tilde{u}\right) \varphi \\
& +2 \rho\left(\log (\bar{\rho}+\tilde{\rho})-\log (\bar{\rho})-\frac{\tilde{\rho}}{\bar{\rho}}\right) \varphi \\
& +\bar{\rho}\left(\nabla_{u} B(\cdot ; \bar{u}+\tilde{u}) u-\nabla_{u} B(\cdot ; \bar{u}) u-\tilde{u}^{T} \nabla_{u u} B(\cdot ; \bar{u}) u\right) \varphi \\
& +\tilde{\rho}\left(\nabla_{u} B(\cdot ; \bar{u}+\tilde{u}) u-\nabla_{u} B(\cdot ; \bar{u}) u\right) \varphi \mathrm{d} \mathbf{x} \\
& \leq\left\|B(\cdot ; \bar{u}+\tilde{u})-B(\cdot ; \bar{u})-\nabla_{u} B(\cdot ; \bar{u})\right\|_{L^{2}\left(W ; \mathbb{R}^{d}\right)}\|\tilde{u}\|_{\mathbb{R}^{\ell}} \\
& +2\left\|\log (\bar{\rho}+\tilde{\rho})-\log (\bar{\rho})-\frac{\tilde{\rho}}{\bar{\rho}}\right\|_{L^{2}(W)} \\
& +\|\bar{\rho}\|_{L^{\infty}(W)}\left\|\nabla_{u} B(\cdot ; \bar{u}+\tilde{u})-\nabla_{u} B(\cdot ; \bar{u})-\tilde{u}^{T} \nabla_{u u} B(\cdot ; \bar{u})\right\|_{L^{2}\left(W ; \mathbb{R}^{d}\right)} \\
& +\|\tilde{\rho}\|_{L^{\infty}(W)}\left\|\nabla_{u} B(\cdot ; \bar{u}+\tilde{u})-\nabla_{u} B(\cdot ; \bar{u})\right\|_{L^{2}\left(W ; \mathbb{R}^{d}\right)} .
\end{aligned}
$$

From (A7) we conclude that

$$
\left\|\nabla_{u} B(\cdot ; \bar{u}+\tilde{u})-\nabla_{u} B(\cdot ; \bar{u})-\tilde{u}^{T} \nabla_{u u} B(\cdot ; \bar{u})\right\|_{L^{2}\left(W ; \mathbb{R}^{d}\right)}=o\left(\|\tilde{u}\|_{\mathbb{R}^{\ell}}\right)
$$

so that there is a constant $c_{8}>0$ depending on $\bar{x}$ such that

$$
\begin{gathered}
\left\|\nabla e^{2}(x+\tilde{x})-\nabla e^{2}(\bar{x})-\nabla^{2} e^{2}(\bar{x})(\tilde{x}, \cdot)\right\|_{L\left(X, H^{\prime}\right)} \\
\leq c_{8}\left(\|\tilde{u}\|_{\mathbb{R}^{\ell}}^{3}+\|\tilde{\rho}\|_{H_{b}}^{2}+\|\tilde{\rho}\|_{L^{\infty}(W)}\|\tilde{u}\|_{\mathbb{R}^{\ell}}\right)+o\left(\|\tilde{u}\|_{\mathbb{R}^{\ell}}\right)=o\left(\|\tilde{x}\|_{X}\right) .
\end{gathered}
$$

Consequently,

$$
\lim _{\|\tilde{x}\|_{X} \rightarrow 0} \frac{\left\|\nabla e^{2}(x+\tilde{x})-\nabla e^{2}(\bar{x})-\nabla^{2} e^{2}(\bar{x})(\tilde{x}, \cdot)\right\|_{L\left(X, H^{\prime}\right)}}{\|\tilde{x}\|_{X}}=0
$$

and the second derivative $\nabla^{2} e^{2}(\bar{x})(\tilde{x}, \cdot)$ is already the second Fréchet-derivative. By (A7) the mapping $u \mapsto \nabla_{u u} B(\cdot ; u)$ is locally Lipschitz-continuous. Hence, $\nabla^{2} e^{2}(\bar{x})(\tilde{x}, \cdot)$ is locally Lipschitz-continuous as well.

Finally we study the operator $e_{3}$. Its directional derivative at the point $\bar{x}$ in any direction $x \in X$ is given by

$$
\left\langle\nabla e^{3}(\bar{x}) x, \varphi\right\rangle_{H_{\circ}^{\prime}, H_{\circ}}=\int_{W} \lambda^{2} \nabla V \cdot \nabla \varphi-2 \bar{\rho} \rho \varphi \mathrm{d} \mathbf{x} \quad \text { for } \varphi \in H_{\circ} .
$$


Then we estimate

$$
\begin{aligned}
& \left\|e^{3}(\bar{x}+x)-e^{3}(\bar{x})-\nabla e^{3}(\bar{x}) x\right\|_{H^{\prime}} \\
\leq & \sup _{\|\varphi\|_{H_{\circ}}=1} \int_{W}\left|\rho^{2} \varphi\right| \mathrm{d} \mathbf{x} \leq \sqrt{\operatorname{meas}(W)}\|\rho\|_{L^{\infty}(W)}^{2} .
\end{aligned}
$$

From (A.11) we directly conclude that $\nabla e_{3}$ is Fréchet-differentiable and the directional derivative coincides with the first Fréchet-derivative. Setting

$$
\left\langle\nabla^{2} e^{3}(\bar{x})(x, \tilde{x}), \varphi\right\rangle_{H_{\circ}^{\prime}, H_{\circ}}=-2 \int_{W} \rho \tilde{\rho} \varphi \mathrm{d} \mathbf{x}
$$

we obtain

$$
\left\|\nabla e^{3}(\bar{x}+\tilde{x})-\nabla e^{3}(\bar{x})-\nabla^{2} e_{3}(\bar{x}) \tilde{x}\right\|_{L\left(X, H^{\prime}\right)}=0
$$

so that $e^{3}$ is twice Fréchet-differentiable with second Fréchet-derivative $\nabla^{2} e^{3}$ given above. Since $\nabla^{2} e^{3}(\bar{x})$ does not depend on $\bar{x}$, the second Fréchet-derivative is Lipschitzcontinuous on $X$.

\section{REFERENCES}

[1] R. A. Adams. Sobolev Spaces, Academic Press, New York, 1975.

[2] M. G. Ancona and G. J. Iafrate, Quantum correction of the equation of state of an electron gas in a semiconductor, Phys. Rev. B, 39, 9536-9540, 1989.

[3] N. Ben Abdallah and A. Unterreiter, On the stationary quantum drift diffusion model, Z. angew. Math. Phys., 49, 251-275, 1998.

[4] M. Burger and R. Pinnau, Fast optimal design of semiconductor devices, SIAM J. Appl. Math., 64, 108-126, 2003.

[5] D. Gilbarg and N. Trudinger, Elliptic Partial Differential Equations of Second Order, SpringerVerlag, Berlin, 1983(2).

[6] M. Hinze and R. Pinnau, Optimal control of the drift diffusion model for semiconductor devices, Hoffmann, K.-H. (ed.) et al., Optimal control of complex structures, Proceedings of the international conference, Oberwolfach, Germany, June 4-10, 2000, Basel: Birkhäuser. ISNM, Int. Ser. Numer. Math. 139, 95-106, 2002.

[7] M. Hinze and R. Pinnau, An optimal control approach to semiconductor design, Math. Models Methods Appl. Sci., 12, 89-107, 2002.

[8] W. Fang and K. Ito, On the stationary semiconductor equations arising in modelling an LBIC technique, Appl. Math. Optim., 33, 189-202, 1996.

[9] K. Ito and K. Kunisch, Newton's method for a class of weakly singular optimal control problems, SIAM J. Optim., 10, 896-916, 2000.

[10] H. Maurer and J. Zowe, First and second order necessary and sufficient optimality conditions for infinite-dimensional programming problems, Mathematical Programming, 16, 98-110, 1979.

[11] F. Pacard and A. Unterreiter, A variational analysis of the thermal equilibrium state of charged quantum fluids, Comm. PDE, 20(5\&6), 885-900,1995.

[12] R. Pinnau, The Quantum Drift Diffusion Model for Semiconductor Devices, PhD Thesis, Department of Mathematics, University of Kaiserslautern, Germany, 2000.

[13] R. Pinnau and A. Unterreiter, The stationary current-voltage characteristics of the quantum drift-diffusion model, SIAM J. Numer. Anal., 37, 211-245, 1999.

[14] M. Reed and B. Simon, Methods of Modern Mathematical Physics I: Functional Analysis, Academic Press, New York, 1980.

[15] M. Stockinger, Optimization of Ultra-Low-Power CMOS Transistors, PhD Thesis, Technical University Vienna, Vienna, Austria, 2000.

[16] M. Stockinger, R. Strasser, R. Plasun, A. Wild and S. Selberherr, A Qualitative Study on Optimized MOSFET Doping Profiles, Proceedings of the Simulation of Semiconductor Processes and Devices (SISPAD 98) Conference, Leuven, Belgium, 1998, Springer-Verlag, New York, 7780, 1998 
[17] M. Stockinger, R. Strasser, R. Plasun, A. Wild and S. Selberherr, Closed-loop MOSFET Doping Profile Optimization for Portable Systems, Proceedings of the International Conference on Modeling and Simulation of Microsystems, Semiconductors, Sensors, and Actuators, San Juan, 1999, Applied Computational Research Society, Cambridge, MA, 411414, 1999.

[18] A. Unterreiter, The thermal equilibrium solution of a generic bipolar hydrodynamic model, Comm. Math. Phys., 188, 69-88, 1997. 